\title{
Dramatic Effects of Halogen Substitution and Solvent on the Rates and Mechanisms of Nucleophilic Substitution Reactions of Aziridines
}

\author{
Harold D. Banks \\ U.S. Army Edgewood Chemical Biological Center, APG, Maryland 21010-5424 \\ harold.banks@us.army.mil
}

Supporting Information Available

\section{Table of Contents}

Energies, Thermal Corrections, Imaginary Frequencies (if any) and Cartesian Coordinates for:

$\mathrm{S}_{\mathrm{N}} 2$ Reactions of $\mathrm{NH}_{3}$ with $\mathbf{1}-\mathbf{1 0}$, cis- and trans-2,3dichloroaziridine in Various Solvents

$\mathrm{S}_{\mathrm{N}} 1$ Reactions of $\mathrm{NH}_{3}$ with 1-7 in Various Solvents

Ground State Energies in Cyclohexane:

$$
\mathrm{S}_{\mathrm{N}} 2
$$

$\mathrm{S}_{\mathrm{N}} 1$

Transition State Data in Cyclohexane:

$$
\mathrm{S}_{\mathrm{N}} 2
$$

$$
\mathrm{S}_{\mathrm{N}} 1
$$

Reaction of Oxirane $+\mathrm{NH}_{3}$ in THF

Atomic Charges and Electrostatic Energies 
Energies (in hartrees) were calculated at the MP2(Full)/6-311++G(G,p)//MP2(Full) $16-31+\mathrm{G}(\mathrm{d})$ level. Data for reactions calculated in the absence of solvent for $\mathbf{2}$ and $\mathbf{3}$ may be found in the Supporting Information of ref 14 .

Aziridine $+\mathrm{NH}_{3}$ Transition State in THF: $\mathrm{E}=-189.9920408$; Thermal Energy Correction $=$ 0.113348 . Unique imaginary frequency $=-686.4797 \mathrm{~cm}^{-1}$.

\begin{tabular}{cccrr} 
Center & Atomic & Coordinates & (Angstroms) \\
Number & Number & $\mathrm{X}$ & \multicolumn{1}{c}{$\mathrm{Y}$} & \multicolumn{1}{c}{$\mathrm{Z}$} \\
-1 & 1 & -0.197655 & -0.999109 & -0.996849 \\
-1 & 6 & -0.137170 & -0.483142 & -0.046687 \\
2 & 1 & -0.167231 & -1.114715 & 0.834414 \\
3 & 6 & 0.791282 & 0.658072 & 0.005044 \\
4 & 7 & -1.958373 & 0.042686 & 0.023914 \\
5 & 7 & 1.925071 & -0.247722 & -0.110996 \\
6 & 1 & 0.711101 & 1.250395 & 0.927885 \\
7 & 1 & 0.700780 & 1.326910 & -0.857595 \\
8 & 1 & -2.591101 & -0.762801 & -0.041381 \\
9 & 1 & -2.169996 & 0.677065 & -0.753372 \\
10 & 1 & -2.159925 & 0.535678 & 0.900080 \\
11 & 1 & 2.182469 & -0.527746 & 0.846251
\end{tabular}

Aziridine in THF: $\mathrm{E}=-133.6214486$; Thermal Energy Correction $=0.074548$.

\begin{tabular}{|c|c|c|c|c|}
\hline Center & Atomic & \multicolumn{3}{|c|}{ Coordinates (Angstroms) } \\
\hline Number & Number & $\mathrm{x}$ & $\mathrm{Y}$ & Z \\
\hline 1 & 6 & 0.741549 & -0.400338 & 0.025135 \\
\hline 2 & 6 & -0.738768 & -0.404424 & 0.025287 \\
\hline 3 & 1 & 1.280882 & -0.753449 & -0.847850 \\
\hline 4 & 1 & 1.251672 & -0.541588 & 0.974372 \\
\hline 5 & 1 & -1.248849 & -0.549572 & 0.973938 \\
\hline 6 & 1 & -1.275889 & -0.759274 & -0.848429 \\
\hline 7 & 7 & -0.002859 & 0.860576 & -0.178036 \\
\hline 8 & 1 & -0.004487 & 1.408420 & 0.691689 \\
\hline
\end{tabular}

$\mathrm{NH}_{3}$ in THF: $\mathrm{E}=-56.4408471$; Thermal Energy Correction $=0.037420$.

\begin{tabular}{ccrrr} 
Center & Atomic & \multicolumn{3}{c}{ Coordinates (Angstroms) } \\
Number & Number & $\mathrm{X}$ & $\mathrm{Y}$ & $\mathrm{Z}$ \\
$-\mathrm{-n}$ & 7 & 0.000000 & 0.000000 & 0.113333 \\
1 & 1 & 0.000000 & 0.947919 & -0.264444 \\
2 & 1 & -0.820922 & -0.473959 & -0.264444 \\
3 & 1 & 0.820922 & -0.473959 & -0.264444
\end{tabular}


Aziridine $+\mathrm{NH}_{3}$ Transition State in MeCN: $\mathrm{E}=-189.9986627$; Thermal Energy Correction $=$ 0.113067 . Unique imaginary frequency $=-716.1149 \mathrm{~cm}^{-1}$.

\begin{tabular}{|c|c|c|c|c|}
\hline \multirow{2}{*}{$\begin{array}{l}\text { Center } \\
\text { Number }\end{array}$} & \multirow{2}{*}{$\begin{array}{l}\text { Atomic } \\
\text { Number }\end{array}$} & \multicolumn{3}{|c|}{ Coordinates (Angstroms) } \\
\hline & & $\mathrm{x}$ & $\mathrm{Y}$ & Z \\
\hline 1 & 1 & -0.193118 & -0.991151 & -1.007493 \\
\hline 2 & 6 & -0.112312 & -0.485904 & -0.053791 \\
\hline 3 & 1 & -0.157061 & -1.112875 & 0.829081 \\
\hline 4 & 6 & 0.791984 & 0.668456 & 0.002532 \\
\hline 5 & 7 & -1.970683 & 0.039625 & 0.026471 \\
\hline 6 & 7 & 1.920252 & -0.251873 & -0.105760 \\
\hline 7 & 1 & 0.711984 & 1.257193 & 0.925950 \\
\hline 8 & 1 & 0.707065 & 1.334375 & -0.861819 \\
\hline 9 & 1 & -2.598662 & -0.769729 & -0.043227 \\
\hline 10 & 1 & -2.192382 & 0.679992 & -0.743691 \\
\hline 11 & 1 & -2.172972 & 0.522640 & 0.908612 \\
\hline 12 & 1 & 2.170134 & -0.530020 & 0.855168 \\
\hline
\end{tabular}

Aziridine in MeCN: $\mathrm{E}=-133.6211706$; Thermal Energy Correction $=0.074398$.

\begin{tabular}{|c|c|c|c|c|}
\hline \multirow{2}{*}{$\begin{array}{l}\text { Center } \\
\text { Number }\end{array}$} & \multirow{2}{*}{$\begin{array}{l}\text { Atomic } \\
\text { Number }\end{array}$} & \multicolumn{3}{|c|}{ Coordinates (Angstroms) } \\
\hline & & $\mathrm{x}$ & $\mathrm{Y}$ & Z \\
\hline 1 & 6 & 0.740278 & -0.402186 & 0.025841 \\
\hline 2 & 6 & -0.739556 & -0.403191 & 0.025813 \\
\hline 3 & 1 & 1.251217 & -0.540731 & 0.975016 \\
\hline 4 & 1 & 1.277211 & -0.759779 & -0.847112 \\
\hline 5 & 1 & -1.250156 & -0.542857 & 0.975049 \\
\hline 6 & 1 & -1.275961 & -0.761888 & -0.847075 \\
\hline 7 & 7 & -0.000734 & 0.861169 & -0.179754 \\
\hline 8 & 1 & -0.001505 & 1.409333 & 0.692478 \\
\hline
\end{tabular}

$\mathrm{NH}_{3}$ in $\mathrm{MeCN}: \mathrm{E}=-56.4421637$; Thermal Energy Correction $=0.037282$.

\begin{tabular}{|c|c|c|c|c|c|}
\hline \multirow{2}{*}{$\begin{array}{l}\text { Center } \\
\text { Number }\end{array}$} & Atomic & & \multicolumn{3}{|c|}{ Coordinates (Angstroms) } \\
\hline & Number & & $\mathrm{x}$ & $\mathrm{Y}$ & Z \\
\hline 1 & 7 & 0 & 0.000000 & 0.000000 & 0.114191 \\
\hline 2 & 1 & 0 & 0.000000 & 0.947693 & -0.266446 \\
\hline 3 & 1 & 0 & -0.820726 & -0.473846 & -0.266446 \\
\hline 4 & 1 & 0 & 0.820726 & -0.473846 & -0.266446 \\
\hline
\end{tabular}


Aziridine $+\mathrm{NH}_{3}$ Transition State in $\mathrm{H}_{2} \mathrm{O}: \mathrm{E}=-189.9997561$; Thermal Energy Correction $=$ 0.113003 . Unique imaginary frequency $=-719.9953 \mathrm{~cm}^{-1}$.

\begin{tabular}{|c|c|c|c|c|c|}
\hline \multirow{2}{*}{$\begin{array}{l}\text { Center } \\
\text { Number }\end{array}$} & \multirow{2}{*}{$\begin{array}{l}\text { Atomic } \\
\text { Number }\end{array}$} & & \multicolumn{3}{|c|}{ Coordinates (Angstroms) } \\
\hline & & & $\mathrm{x}$ & $\mathrm{Y}$ & Z \\
\hline 1 & 1 & 0 & -0.192232 & -0.992437 & -1.005717 \\
\hline 2 & 6 & 0 & -0.108140 & -0.487276 & -0.052405 \\
\hline 3 & 1 & 0 & -0.155503 & -1.111831 & 0.831941 \\
\hline 4 & 6 & 0 & 0.791463 & 0.669789 & 0.002821 \\
\hline 5 & 7 & 0 & -1.972895 & 0.039261 & 0.025962 \\
\hline 6 & 7 & 0 & 1.919725 & -0.251918 & -0.106877 \\
\hline 7 & 1 & 0 & 0.713024 & 1.257470 & 0.926722 \\
\hline 8 & 1 & 0 & -2.600973 & -0.771167 & -0.029055 \\
\hline 9 & 1 & 0 & -2.196406 & 0.667256 & -0.753895 \\
\hline 10 & 1 & 0 & -2.172540 & 0.536377 & 0.900908 \\
\hline 11 & 1 & 0 & 2.171930 & -0.527638 & 0.854401 \\
\hline 12 & 1 & 0 & 0.704953 & 1.335494 & -0.861397 \\
\hline
\end{tabular}

Aziridine in $\mathrm{H}_{2} \mathrm{O}$ : $\mathrm{E}=-133.6214486$; Thermal Energy Correction $=0.074548$.

\begin{tabular}{|c|c|c|c|c|c|}
\hline Center & Atomic & & Coorc & nates & roms ) \\
\hline Number & Number & & $\mathrm{X}$ & Y & Z \\
\hline 1 & 6 & 0 & 0.740057 & -0.402477 & 0.025874 \\
\hline 2 & 6 & 0 & -0.739844 & -0.402700 & 0.025775 \\
\hline 3 & 1 & 0 & 1.250903 & -0.540352 & 0.975262 \\
\hline 4 & 1 & 0 & 1.277239 & -0.760020 & -0.847044 \\
\hline 5 & 1 & 0 & -1.250199 & -0.542231 & 0.975159 \\
\hline 6 & 1 & 0 & -1.276350 & -0.761157 & -0.847152 \\
\hline 7 & 7 & 0 & -0.000215 & 0.860964 & -0.179920 \\
\hline 8 & 1 & 0 & -0.001364 & 1.408071 & 0.693325 \\
\hline
\end{tabular}

$\mathrm{NH}_{3}$ in $\mathrm{H}_{2} \mathrm{O}: \quad \mathrm{E}=-56.4423619$; Thermal Energy Correction $=0.037248$.

\begin{tabular}{|c|c|c|c|c|c|}
\hline \multirow{2}{*}{$\begin{array}{l}\text { Center } \\
\text { Number }\end{array}$} & \multirow{2}{*}{$\begin{array}{l}\text { Atomic } \\
\text { Number }\end{array}$} & \multirow{2}{*}{$\begin{array}{l}\text { Atomic } \\
\text { Type }\end{array}$} & \multicolumn{3}{|c|}{ Coordinates (Angstroms) } \\
\hline & & & $\mathrm{x}$ & $\mathrm{Y}$ & $\mathrm{Z}$ \\
\hline 1 & 7 & 0 & 0.000000 & 0.000000 & 0.114730 \\
\hline 2 & 1 & 0 & 0.000000 & 0.947472 & -0.267703 \\
\hline 3 & 1 & 0 & -0.820535 & -0.473736 & -0.267703 \\
\hline 4 & 1 & 0 & 0.820535 & -0.473736 & -0.267703 \\
\hline
\end{tabular}


2-Fluoroaziridine $+\mathrm{NH}_{3}$ Transition State in THF: $\mathrm{E}=-289.1138630$; Thermal Energy Correction $=0.105308$. Unique imaginary frequency $=-678.6912 \mathrm{~cm}^{-1}$.

\begin{tabular}{|c|c|c|c|c|}
\hline \multirow{2}{*}{$\begin{array}{l}\text { Center } \\
\text { Number }\end{array}$} & \multirow{2}{*}{$\begin{array}{l}\text { Atomic } \\
\text { Number }\end{array}$} & \multicolumn{3}{|c|}{ Coordinates (Angstroms) } \\
\hline & & $\mathrm{x}$ & $\mathrm{Y}$ & Z \\
\hline 1 & 1 & -0.321475 & -0.931144 & -1.221596 \\
\hline 2 & 6 & -0.264318 & -0.847370 & -0.145530 \\
\hline 3 & 1 & -0.506634 & -1.717288 & 0.452231 \\
\hline 4 & 6 & 0.722040 & 0.077157 & 0.380958 \\
\hline 5 & 7 & -2.105107 & -0.048017 & 0.032363 \\
\hline 6 & 7 & 1.702793 & -0.692060 & -0.222218 \\
\hline 7 & 9 & 0.567774 & 1.437194 & -0.162719 \\
\hline 8 & 1 & -2.358452 & 0.102722 & 1.013502 \\
\hline 9 & 1 & 0.741303 & 0.221895 & 1.465910 \\
\hline 10 & 1 & -2.848130 & -0.603241 & -0.404508 \\
\hline 11 & 1 & -2.074915 & 0.865539 & -0.428884 \\
\hline 12 & 1 & 2.328203 & -1.071403 & 0.504233 \\
\hline
\end{tabular}

2-Fluoroaziridine $+\mathrm{NH}_{3}$ Transition State in $\mathrm{MeCN}: \mathrm{E}=-289.1194977$; Thermal Energy Correction $=0.104928$. Unique imaginary frequency $=-689.7674 \mathrm{~cm}^{-1}$.

\begin{tabular}{cccrrr}
$\begin{array}{c}\text { Center } \\
\text { Number }\end{array}$ & $\begin{array}{c}\text { Atomic } \\
\text { Number }\end{array}$ & $\begin{array}{c}\text { Atomic } \\
\text { Type }\end{array}$ & \multicolumn{2}{c}{ Coordinates (Angstroms) } \\
$-0-1$ & 0 & -0.336281 & -0.888271 & -1.241161 \\
1 & 1 & 0 & -0.267945 & -0.830635 & -0.164215 \\
2 & 6 & 0 & -0.524100 & -1.705222 & 0.420282 \\
3 & 1 & 0 & 0.720004 & 0.077314 & 0.384079 \\
4 & 6 & 0 & -2.125243 & -0.033858 & 0.036980 \\
5 & 7 & 0 & 1.691259 & -0.716617 & -0.212317 \\
6 & 7 & 0 & 0.616016 & 1.434633 & -0.164716 \\
7 & 9 & 0 & -2.360687 & 0.115722 & 1.023522 \\
8 & 1 & 0 & 0.729615 & 0.216897 & 1.469703 \\
9 & 1 & 0 & -2.864274 & -0.611690 & -0.378462 \\
10 & 1 & 0 & -2.134931 & 0.877799 & -0.430389 \\
11 & 1 & 0 & 2.272041 & -1.143680 & 0.527110 \\
12 & 1 & & & &
\end{tabular}

2-Fluoroaziridine $+\mathrm{NH}_{3}$ Transition State in $\mathrm{H}_{2} \mathrm{O}: \mathrm{E}=-289.1204586$; Thermal Energy Correction $=0.104843$. Unique imaginary frequency $=-692.4591 \mathrm{~cm}^{-1}$.

\begin{tabular}{cccrr} 
Center & Atomic & \multicolumn{3}{c}{ Coordinates (Angstroms) } \\
Number & Number & \multicolumn{1}{c}{ X } & \multicolumn{1}{c}{ Y } \\
-1 & 1 & -0.337110 & -0.882355 & -1.245273 \\
-1 & 6 & -0.267071 & -0.829719 & -0.168181 \\
3 & 1 & -0.524897 & -1.705486 & 0.413691 \\
4 & 6 & 0.718668 & 0.077436 & 0.384672 \\
5 & 7 & -2.127074 & -0.032825 & 0.038017 \\
6 & 7 & 1.690410 & -0.718317 & -0.209998 \\
7 & 1 & 0.725492 & 0.216511 & 1.470333 \\
8 & 1 & -2.866257 & -0.604110 & -0.386303 \\
9 & 1 & -2.134257 & 0.884962 & -0.417546
\end{tabular}




$\begin{array}{rrrrr}10 & 1 & -2.364114 & 0.104232 & 1.026096 \\ 11 & 1 & 2.264723 & -1.151259 & 0.531470 \\ 12 & 9 & 0.620387 & 1.434355 & -0.165284\end{array}$

2-Fluoroaziridine in THF: $E=-232.7204044$; Thermal Energy Correction $=0.066528$.

\begin{tabular}{ccrrr} 
Center & Atomic & \multicolumn{3}{c}{ Coordinates (Angstroms) } \\
Number & Number & $\mathrm{X}$ & $\mathrm{Y}$ & \multicolumn{1}{c}{$\mathrm{Z}$} \\
-1 & 6 & -0.946293 & -0.658204 & -0.129664 \\
2 & 6 & 0.211484 & -0.017521 & 0.500584 \\
3 & 1 & -0.769650 & -1.119236 & -1.097829 \\
4 & 1 & -1.741505 & -1.088622 & 0.470260 \\
5 & 7 & -0.812306 & 0.830572 & -0.038180 \\
6 & 1 & -0.517469 & 1.224968 & -0.944275 \\
7 & 9 & 1.411787 & -0.088418 & -0.216999 \\
8 & 1 & 0.417537 & 0.019001 & 1.566580
\end{tabular}

2-Fluoroaziridine in MeCN: $\mathrm{E}=-232.7257155$; Thermal Energy Correction $=0.066427$.

\begin{tabular}{|c|c|c|c|c|}
\hline \multirow{2}{*}{$\begin{array}{l}\text { Center } \\
\text { Number }\end{array}$} & \multirow{2}{*}{$\begin{array}{l}\text { Atomic } \\
\text { Number }\end{array}$} & \multicolumn{3}{|c|}{ Coordinates (Angstroms) } \\
\hline & & $\mathrm{X}$ & $\mathrm{Y}$ & Z \\
\hline 1 & 6 & -0.214009 & -0.078187 & 0.451065 \\
\hline 2 & 6 & 0.923703 & 0.708354 & -0.030573 \\
\hline 3 & 1 & 0.787263 & 1.357784 & -0.889082 \\
\hline 4 & 1 & 1.683178 & 1.009055 & 0.686880 \\
\hline 5 & 7 & 0.763188 & -0.744426 & -0.345776 \\
\hline 6 & 1 & 1.370410 & -1.350238 & 0.229435 \\
\hline 7 & 1 & -0.360883 & -0.334519 & 1.498100 \\
\hline 8 & 9 & -1.453383 & 0.083100 & -0.180873 \\
\hline
\end{tabular}

2-Fluoroaziridine in $\mathrm{H}_{2} \mathrm{O}$ : $\mathrm{E}=-232.7226827$; Thermal Energy Correction $=0.066248$.

\begin{tabular}{rrrrr} 
Center & Atomic & \multicolumn{3}{c}{ Coordinates } \\
Number & Number & X & Y & $Z$ \\
$-r-1$ & 1 & -0.337110 & -0.882355 & -1.245273 \\
1 & 6 & -0.267071 & -0.829719 & -0.168181 \\
2 & 1 & -0.524897 & -1.705486 & 0.413691 \\
3 & 6 & 0.718668 & 0.077436 & 0.384672 \\
4 & 7 & -2.127074 & -0.032825 & 0.038017 \\
5 & 7 & 1.690410 & -0.718317 & -0.209998 \\
6 & 1 & 0.725492 & 0.216511 & 1.470333 \\
7 & 1 & -2.866257 & -0.604110 & -0.386303 \\
8 & 1 & -2.134257 & 0.884962 & -0.417546 \\
9 & 1 & -2.364114 & 0.104232 & 1.026096 \\
10 & 1 & 2.264723 & -1.151259 & 0.531470 \\
11 & 9 & 0.620387 & 1.434355 & -0.165284 \\
12 & & & &
\end{tabular}


2,2-Difluoroaziridine $+\mathrm{NH}_{3}$ Transition State in THF: $\mathrm{E}=-388.2325246$; Thermal Energy Correction $=0.097093$. Unique imaginary frequency $=-595.6432 \mathrm{~cm}^{-1}$.

\begin{tabular}{|c|c|c|c|c|}
\hline Center & Atomic & Coorc & hates (Angs & roms ) \\
\hline Number & Number & $\mathrm{X}$ & $Y$ & Z \\
\hline 1 & 1 & 0.670136 & 1.373599 & -1.060287 \\
\hline 2 & 6 & 0.452167 & 0.949843 & -0.090425 \\
\hline 3 & 1 & 0.634983 & 1.537754 & 0.799393 \\
\hline 4 & 6 & -0.617041 & -0.018082 & -0.022842 \\
\hline 5 & 7 & 2.258097 & -0.023325 & 0.033049 \\
\hline 6 & 7 & -1.544164 & 0.965939 & -0.187486 \\
\hline 7 & 9 & -0.596784 & -0.977005 & -1.041434 \\
\hline 8 & 1 & 2.306233 & -0.548886 & 0.911167 \\
\hline 9 & 9 & -0.642969 & -0.772036 & 1.166075 \\
\hline 10 & 1 & 3.074631 & 0.595187 & -0.012046 \\
\hline 11 & 1 & 2.325181 & -0.693351 & -0.739029 \\
\hline 12 & 1 & -1.861672 & 1.288204 & 0.739696 \\
\hline
\end{tabular}

2,2-Difluoroaziridine $+\mathrm{NH}_{3}$ Transition State in $\mathrm{MeCN}: \mathrm{E}=-388.2375514$; Thermal Energy Correction $=0.096707$. Unique imaginary frequency $=-607.4049 \mathrm{~cm}^{-1}$.

\begin{tabular}{|c|c|c|c|c|}
\hline Center & Atomic & Coorc & hates (Angs & roms ) \\
\hline Number & Number & $\mathrm{X}$ & $\mathrm{Y}$ & Z \\
\hline 1 & 1 & 0.677162 & 1.357055 & -1.070134 \\
\hline 2 & 6 & 0.445673 & 0.948771 & -0.096883 \\
\hline 3 & 1 & 0.645451 & 1.532356 & 0.792004 \\
\hline 4 & 6 & -0.618408 & -0.020297 & -0.019831 \\
\hline 5 & 7 & 2.277814 & -0.032188 & 0.030768 \\
\hline 6 & 7 & -1.532221 & 0.981477 & -0.173029 \\
\hline 7 & 9 & -0.625542 & -0.971515 & -1.044912 \\
\hline 8 & 1 & 2.342050 & -0.544387 & 0.916371 \\
\hline 9 & 9 & -0.648409 & -0.780407 & 1.162167 \\
\hline 10 & 1 & 3.083373 & 0.600537 & -0.026027 \\
\hline 11 & 1 & 2.357747 & -0.711893 & -0.732402 \\
\hline 12 & 1 & -1.822959 & 1.317766 & 0.761006 \\
\hline
\end{tabular}

2,2-Difluoroaziridine $+\mathrm{NH}_{3}$ Transition State in $\mathrm{H}_{2} \mathrm{O}: \quad \mathrm{E}=-388.2383935$; Thermal Energy Correction $=0.096619$. Unique imaginary frequency $=-609.3275 \mathrm{~cm}^{-1}$.

\begin{tabular}{rrrrr} 
Center & Atomic & \multicolumn{3}{c}{ Coordinates (Angstroms) } \\
Number & Number & \multicolumn{1}{c}{ X } & $Z$ \\
$-r$ & 1 & 0.679639 & 1.354672 & -1.070107 \\
1 & 6 & 0.445211 & 0.948537 & -0.096678 \\
3 & 1 & 0.647265 & 1.530823 & 0.792558 \\
4 & 6 & -0.618450 & -0.020387 & -0.019428 \\
5 & 7 & 2.280940 & -0.034044 & 0.030824 \\
6 & 7 & -1.529171 & 0.984547 & -0.173046 \\
7 & 1 & 3.084958 & 0.600706 & -0.026939 \\
8 & 1 & 2.361992 & -0.713868 & -0.732245 \\
9 & 1 & 2.347158 & -0.545569 & 0.916773 \\
10 & 1 & -1.818608 & 1.321734 & 0.761579 \\
11 & 9 & -0.628946 & -0.971404 & -1.045058 \\
12 & 9 & -0.651649 & -0.780920 & 1.161788
\end{tabular}


2,2-Difluoroaziridine in THF: $E=-331.8257475$; Thermal Energy Correction $=0.058734$.

\begin{tabular}{ccrrr} 
Center & Atomic & \multicolumn{3}{c}{ Coordinates (Angstroms) } \\
Number & Number & $\mathrm{X}$ & $\mathrm{Y}$ & $\mathrm{Z}$ \\
-1 & 6 & -1.158836 & -0.123410 & -0.662952 \\
2 & 6 & 0.140098 & -0.015302 & -0.023001 \\
3 & 1 & -1.559417 & 0.770027 & -1.134760 \\
4 & 1 & -1.506238 & -1.080499 & -1.037183 \\
5 & 7 & -0.950790 & -0.093012 & 0.849520 \\
6 & 1 & -1.190217 & 0.824282 & 1.259298 \\
7 & 9 & 0.853154 & 1.149708 & -0.110601 \\
8 & 9 & 1.038382 & -1.041981 & 0.008570
\end{tabular}

2,2-Difluoroaziridine in MeCN: $\mathrm{E}=-331.8279122$; Thermal Energy Correction $=0.058481$.

\begin{tabular}{|c|c|c|c|c|}
\hline Center & Atomic & Coord & ates (Angst & oms ) \\
\hline Number & Number & X & $\mathrm{Y}$ & z \\
\hline 1 & 6 & -0.138329 & -0.013835 & -0.023070 \\
\hline 2 & 6 & 1.159713 & -0.123205 & -0.663360 \\
\hline 3 & 1 & 1.504480 & -1.080836 & -1.039603 \\
\hline 4 & 1 & 1.562769 & 0.770743 & -1.132567 \\
\hline 5 & 7 & 0.950878 & -0.092169 & 0.849975 \\
\hline 6 & 1 & 1.192964 & 0.828691 & 1.259220 \\
\hline 7 & 9 & -1.037734 & -1.042872 & 0.009313 \\
\hline 8 & 9 & -0.856117 & 1.148297 & -0.111345 \\
\hline
\end{tabular}

2,2-Difluoroaziridine in $\mathrm{H}_{2} \mathrm{O}: \quad \mathrm{E}=-331.8283221$; Thermal Energy Correction $=0.058426$.

\begin{tabular}{|c|c|c|c|c|}
\hline \multirow{2}{*}{$\begin{array}{l}\text { Center } \\
\text { Number }\end{array}$} & \multirow{2}{*}{$\begin{array}{l}\text { Atomic } \\
\text { Number }\end{array}$} & \multicolumn{3}{|c|}{ Coordinates (Angstroms) } \\
\hline & & $\mathrm{x}$ & $\mathrm{Y}$ & z \\
\hline 1 & 6 & -0.138080 & -0.013640 & -0.023220 \\
\hline 2 & 6 & 1.159681 & -0.124145 & -0.663011 \\
\hline 3 & 1 & 1.503045 & -1.082397 & -1.039143 \\
\hline 4 & 1 & 1.563894 & 0.768212 & -1.134289 \\
\hline 5 & 7 & 0.950996 & -0.091075 & 0.850720 \\
\hline 6 & 1 & 1.190679 & 0.832949 & 1.255716 \\
\hline 7 & 9 & -1.037540 & -1.043246 & 0.009956 \\
\hline 8 & 9 & -0.856260 & 1.148298 & -0.112172 \\
\hline
\end{tabular}


2-Chloroaziridine $+\mathrm{NH}_{3}$ Transition State (No Solvent): $\mathrm{E}=-649.0984237$; Thermal Energy Correction $=0.105752$. Unique imaginary frequency $=-669.4331 \mathrm{~cm}^{-1}$.

\begin{tabular}{cccrr} 
Center & Atomic & \multicolumn{3}{c}{ Coordinates (Angstroms) } \\
Number & Number & $\mathrm{X}$ & $\mathrm{Y}$ & $\mathrm{Z}$ \\
--1 & 1 & 0.730920 & 1.183921 & -1.212038 \\
1 & 6 & 0.680984 & 1.123194 & -0.134107 \\
2 & 1 & 1.188117 & 1.892069 & 0.438156 \\
3 & 6 & -0.542912 & 0.569904 & 0.415682 \\
4 & 7 & -1.259700 & 1.499880 & -0.271866 \\
5 & 1 & -1.901234 & 1.998260 & 0.356027 \\
6 & 7 & 2.130124 & -0.238932 & 0.021358 \\
7 & 1 & 2.496544 & -0.355571 & 0.966512 \\
8 & 1 & 2.914148 & -0.132322 & -0.623459 \\
9 & 1 & 1.593350 & -1.077247 & -0.219912 \\
10 & 17 & -0.784389 & -1.352283 & -0.067329 \\
11 & 1 & -0.608633 & 0.494484 & 1.503415 \\
12 & & & &
\end{tabular}

2-Chloroaziridine $+\mathrm{NH}_{3}$ Transition State in THF: $\mathrm{E}=-649.1216581$; Thermal Energy Correction $=0.104425$. Unique imaginary frequency $=-666.9867 \mathrm{~cm}^{-1}$.

\begin{tabular}{ccrrr} 
Center & Atomic & \multicolumn{3}{c}{ Coordinates (Angstroms) } \\
Number & Number & \multicolumn{1}{c}{ X } & \multicolumn{1}{c}{$\mathrm{Z}$} \\
-1 & 6 & -0.744427 & 0.992308 & -0.170553 \\
-1 & 6 & 0.478011 & 0.539462 & 0.459820 \\
2 & 7 & -2.224516 & -0.409062 & 0.009435 \\
3 & 7 & 1.168671 & 1.633701 & -0.039328 \\
4 & 1 & -3.156010 & -0.048494 & -0.221938 \\
5 & 1 & -2.249939 & -0.754686 & 0.973519 \\
6 & 1 & -2.028735 & -1.206396 & -0.602135 \\
7 & 1 & 1.268777 & 1.555503 & -1.064266 \\
8 & 1 & -1.321499 & 1.745807 & 0.350856 \\
9 & 1 & -0.786659 & 0.980597 & -1.252916 \\
10 & 17 & 0.987838 & -1.207128 & -0.074077 \\
11 & 1 & 0.470223 & 0.485744 & 1.549830 \\
12 & & & &
\end{tabular}

2-Chloroaziridine $+\mathrm{NH}_{3}$ Transition State in $\mathrm{MeCN}: \mathrm{E}=-649.1265195$; Thermal Energy Correction $=0.104070$. Unique imaginary frequency $=-680.9486 \mathrm{~cm}^{-1}$.

\begin{tabular}{rrrrr} 
Center & Atomic & \multicolumn{3}{c}{ Coordinates (Angstroms) } \\
Number & Number & $X$ & $Y$ & $Z$ \\
-1 & 6 & -0.747810 & 0.981304 & -0.174873 \\
1 & 6 & 0.469641 & 0.528600 & 0.463787 \\
2 & 7 & -2.238081 & -0.430624 & 0.008761 \\
3 & 7 & 1.138292 & 1.643537 & -0.036483 \\
4 & 1 & -3.168319 & -0.051174 & -0.199254 \\
5 & 1 & -2.253071 & -0.790038 & 0.968736 \\
6 & 1 & -2.071444 & -1.223075 & -0.618887 \\
7 & 1 & 1.264016 & 1.552872 & -1.059155 \\
8 & 1 & -1.336657 & 1.725114 & 0.346454 \\
9 & 1 & -0.792793 & 0.957405 & -1.256706 \\
10 & 17 & 1.015954 & -1.188012 & -0.074991 \\
11 & & & &
\end{tabular}



12
1
0.454588
0.475290
1.554231

2-Chloroaziridine $+\mathrm{NH}_{3}$ Transition State in $\mathrm{H}_{2} \mathrm{O}: \mathrm{E}=-649.1274020$; Thermal Energy Correction $=0.103970$. Unique imaginary frequency $=-683.8177 \mathrm{~cm}^{-1}$.

\begin{tabular}{rrrrr} 
Center & Atomic & \multicolumn{3}{c}{ Coordinates (Angstroms) } \\
Number & Number & $\mathrm{X}$ & $\mathrm{Y}$ & $\mathrm{Z}$ \\
$-0-1$ & 6 & -0.746725 & 0.982081 & -0.175288 \\
1 & 6 & 0.468747 & 0.526994 & 0.464771 \\
2 & 7 & -2.239493 & -0.432529 & 0.008585 \\
3 & 7 & 1.135565 & 1.643872 & -0.036388 \\
4 & 1 & -3.168147 & -0.058445 & -0.215773 \\
5 & 1 & -2.265250 & -0.779781 & 0.972860 \\
6 & 1 & -2.062312 & -1.232287 & -0.607016 \\
7 & 1 & 1.262176 & 1.550623 & -1.059213 \\
8 & 1 & -1.337569 & 1.724062 & 0.346321 \\
9 & 1 & -0.792588 & 0.955695 & -1.256967 \\
10 & 17 & 1.018013 & -1.186323 & -0.075161 \\
11 & 1 & 0.452827 & 0.473766 & 1.555249 \\
12 & & & &
\end{tabular}

2-Chloroaziridine $+\mathrm{NH}_{3}$ Dipolar Complex (No Solvent): ): $\mathrm{E}=-649.1644480$; Thermal Energy Correction $=0.107826$.

\begin{tabular}{|c|c|c|c|c|}
\hline Center & Atomic & Coorc & $\begin{array}{l}\text { (Angs } \\
\mathrm{Y}\end{array}$ & roms ) \\
\hline & Number & & $\mathrm{Y}$ & Z \\
\hline 1 & 6 & 1.035215 & 0.576388 & -0.043147 \\
\hline 2 & 6 & 0.050972 & 1.353791 & 0.730203 \\
\hline 3 & 1 & -0.719322 & 0.829774 & 1.285992 \\
\hline 4 & 1 & 0.365241 & 2.318531 & 1.121169 \\
\hline 5 & 7 & -0.082474 & 1.181348 & -0.738281 \\
\hline 6 & 1 & 0.212150 & 2.004091 & -1.267172 \\
\hline 7 & 1 & -3.548131 & -0.449606 & -0.245892 \\
\hline 8 & 7 & -2.589562 & -0.554231 & 0.079958 \\
\hline 9 & 1 & -2.362211 & -1.545787 & 0.047483 \\
\hline 10 & 1 & -1.974837 & -0.078146 & -0.581634 \\
\hline 11 & 17 & 1.069503 & -1.177917 & 0.019103 \\
\hline 12 & 1 & 2.032685 & 0.974840 & -0.198764 \\
\hline
\end{tabular}

2-Chloroaziridine in THF: $E=-592.7309660$; Thermal Energy Correction $=0.065758$.

\begin{tabular}{|c|c|c|c|c|}
\hline Center & Atomic & Coord & nates & roms) \\
\hline Number & Number & $\mathrm{X}$ & $\mathrm{Y}$ & Z \\
\hline 1 & 6 & 0.266585 & -0.109707 & 0.509689 \\
\hline 2 & 6 & 1.340371 & 0.724962 & -0.054341 \\
\hline 3 & 1 & 1.113498 & 1.392604 & -0.879415 \\
\hline 4 & 1 & 2.138686 & 1.042246 & 0.612435 \\
\hline 5 & 7 & 1.219202 & -0.715431 & -0.392129 \\
\hline 6 & 1 & 1.892071 & -1.295747 & 0.128991 \\
\hline 7 & 17 & -1.388092 & 0.032719 & -0.083080 \\
\hline 8 & 1 & 0.277155 & -0.378845 & 1.563162 \\
\hline
\end{tabular}


2-Chloroaziridine in MeCN: $\mathrm{E}=-592.7333528$; Thermal Energy Correction $=0.065758$.

\begin{tabular}{|c|c|c|c|c|}
\hline Center & Atomic & Coor & hates (Angs & roms ) \\
\hline Number & Number & $\mathrm{x}$ & $\mathrm{Y}$ & Z \\
\hline 1 & 6 & 0.266585 & -0.109707 & 0.509689 \\
\hline 2 & 6 & 1.340371 & 0.724962 & -0.054341 \\
\hline 3 & 1 & 1.113498 & 1.392604 & -0.879415 \\
\hline 4 & 1 & 2.138686 & 1.042246 & 0.612435 \\
\hline 5 & 7 & 1.219202 & -0.715431 & -0.392129 \\
\hline 6 & 1 & 1.892071 & -1.295747 & 0.128991 \\
\hline 7 & 17 & -1.388092 & 0.032719 & -0.083080 \\
\hline 8 & 1 & 0.277155 & -0.378846 & 1563162 \\
\hline
\end{tabular}

2-Chloroaziridine in $\mathrm{H}_{2} \mathrm{O}: \quad \mathrm{E}=-592.7337508$; Thermal Energy Correction $=0.065488$.

\begin{tabular}{|c|c|c|c|c|}
\hline Center & Atomic & \multicolumn{3}{|c|}{ Coordinates (Angstroms) } \\
\hline Number & Number & $\mathrm{X}$ & $\mathrm{Y}$ & Z \\
\hline 1 & 6 & 0.268545 & -0.111798 & 0.510213 \\
\hline 2 & 6 & 1.339653 & 0.726118 & -0.052961 \\
\hline 3 & 1 & 1.110536 & 1.397306 & -0.874933 \\
\hline 4 & 1 & 2.139662 & 1.039950 & 0.613555 \\
\hline 5 & 7 & 1.219726 & -0.714066 & -0.395150 \\
\hline 6 & 1 & 1.896488 & -1.294335 & 0.128562 \\
\hline 7 & 17 & -1.389044 & 0.032755 & -0.082798 \\
\hline 8 & 1 & 0.279791 & -0.387209 & 1.562910 \\
\hline
\end{tabular}

2,2-Dichloroaziridine $+\mathrm{NH}_{3}$ Transition State (No Solvent): $\mathrm{E}=-1108.2150794$; Thermal Energy Correction $=0.096571$. Unique imaginary frequency $=-663.0198 \mathrm{~cm}^{-1}$.

\begin{tabular}{|c|c|c|c|c|}
\hline Center & Atomic & Coorc & hates (Angs & roms ) \\
\hline Number & Number & $\mathrm{X}$ & $\mathrm{Y}$ & Z \\
\hline 1 & 1 & 1.295269 & -0.241751 & 1.778016 \\
\hline 2 & 6 & 0.668213 & -0.733114 & 1.046924 \\
\hline 3 & 1 & 0.447678 & -1.785543 & 1.176981 \\
\hline 4 & 6 & -0.380895 & 0.082713 & 0.453446 \\
\hline 5 & 7 & 2.074315 & -0.975641 & -0.313523 \\
\hline 6 & 7 & -0.951184 & 0.249686 & 1.659345 \\
\hline 7 & 1 & 2.918179 & -1.477772 & -0.031990 \\
\hline 8 & 1 & 2.317105 & -0.023493 & -0.594818 \\
\hline 9 & 1 & 1.661197 & -1.443806 & -1.121933 \\
\hline 10 & 1 & -1.904546 & -0.118803 & 1.711156 \\
\hline 11 & 17 & 0.316173 & 1.646504 & -0.407482 \\
\hline 12 & 17 & -1.276214 & -0.818548 & -0.847835 \\
\hline
\end{tabular}


2,2-Dichloroaziridine $+\mathrm{NH}_{3}$ Transition State in THF: $\mathrm{E}=-1108.2354010$; Thermal Energy Correction $=0.095016$. Unique imaginary frequency $=-645.3002 \mathrm{~cm}^{-1}$.

\begin{tabular}{rrrrr} 
Center & Atomic & \multicolumn{3}{c}{ Coordinates (Angstroms) } \\
Number & Number & \multicolumn{1}{c}{ X } & \multicolumn{1}{c}{ Y } & $\mathrm{Z}$ \\
-1 & 6 & 0.912039 & -0.207391 & 1.052663 \\
-1 & 6 & -0.374724 & 0.019046 & 0.419346 \\
2 & 7 & 2.383343 & -0.243492 & -0.325181 \\
3 & 7 & -0.954113 & 0.071698 & 1.657531 \\
4 & 1 & 3.286403 & -0.493714 & 0.092814 \\
5 & 1 & 2.483333 & 0.676656 & -0.764651 \\
6 & 1 & 2.179562 & -0.923605 & -1.063960 \\
7 & 1 & -1.399804 & -0.827576 & 1.897062 \\
8 & 1 & 1.344830 & 0.626474 & 1.588081 \\
9 & 1 & 1.113767 & -1.215671 & 1.392919 \\
10 & 17 & -0.855409 & -1.323062 & -0.718213 \\
11 & 17 & -0.452626 & 1.587184 & -0.534773 \\
12 & & & &
\end{tabular}

2,2-Dichloroaziridine $+\mathrm{NH}_{3}$ Transition State in MeCN: $\mathrm{E}=-1108.2400343$; Thermal Energy Correction $=0.093746$. Unique imaginary frequency $=-652.0966 \mathrm{~cm}^{-1}$.

\begin{tabular}{|c|c|c|c|c|}
\hline Center & Atomic & Coorc & nates (Angs & roms ) \\
\hline Number & Number & $\mathrm{x}$ & $\mathrm{Y}$ & z \\
\hline 1 & 1 & 1.281643 & 0.868654 & 1.512972 \\
\hline 2 & 6 & 0.927626 & -0.043314 & 1.052148 \\
\hline 3 & 1 & 1.241130 & -0.999252 & 1.453077 \\
\hline 4 & 6 & -0.372448 & 0.001969 & 0.411114 \\
\hline 5 & 7 & 2.417399 & -0.033238 & -0.335969 \\
\hline 6 & 7 & -0.948699 & 0.031340 & 1.658103 \\
\hline 7 & 1 & 3.336586 & -0.059069 & 0.120931 \\
\hline 8 & 1 & 2.378944 & 0.813905 & -0.912306 \\
\hline 9 & 1 & 2.357320 & -0.842696 & -0.962430 \\
\hline 10 & 1 & -1.195321 & -0.925883 & 1.964045 \\
\hline 11 & 17 & -0.652095 & 1.509755 & -0.571801 \\
\hline 12 & 17 & -0.701568 & -1.427067 & -0.675893 \\
\hline
\end{tabular}

2,2-Dichloroaziridine $+\mathrm{NH}_{3}$ Transition State in $\mathrm{H}_{2} \mathrm{O}: \quad \mathrm{E}=-1$ 108.2409522; Thermal Energy Correction $=0.093645$. Unique imaginary frequency $=-655.8922 \mathrm{~cm}^{-1}$.

\begin{tabular}{|c|c|c|c|c|}
\hline $\begin{array}{l}\text { Center } \\
\text { Number }\end{array}$ & $\begin{array}{l}\text { Atomic } \\
\text { Number }\end{array}$ & $\begin{array}{l}\text { Coord: } \\
x\end{array}$ & $\begin{array}{cl}\text { ates (Angs } \\
y \\
y\end{array}$ & oms ) \\
\hline 1 & 1 & 1.276591 & 0.880812 & 1.510308 \\
\hline 2 & 6 & 0.925642 & -0.035092 & 1.055093 \\
\hline 3 & 1 & 1.245643 & -0.988079 & 1.457797 \\
\hline 4 & 6 & -0.372200 & 0.000726 & 0.409912 \\
\hline 5 & 7 & 2.418138 & -0.022374 & -0.336544 \\
\hline 6 & 7 & -0.948660 & 0.028763 & 1.657962 \\
\hline 7 & 1 & 3.338946 & -0.031052 & 0.117715 \\
\hline 8 & 1 & 2.365709 & 0.817725 & -0.922312 \\
\hline 9 & 1 & 2.365222 & -0.840006 & -0.953222 \\
\hline 10 & 1 & -1.183581 & -0.931443 & 1.965655 \\
\hline 11 & 17 & -0.661409 & 1.505187 & -0.573636 \\
\hline 12 & 17 & -0.692445 & -1.431451 & -0.674358 \\
\hline
\end{tabular}


2,2-Dichloroaziridine $+\mathrm{NH}_{3}$ Dipolar Complex (No Solvent): $\mathrm{E}=-1108.2698036$; Thermal Energy Correction $=0.098783$.

\begin{tabular}{|c|c|c|c|c|}
\hline \multirow{2}{*}{$\begin{array}{l}\text { Center } \\
\text { Number }\end{array}$} & \multirow{2}{*}{$\begin{array}{l}\text { Atomic } \\
\text { Number }\end{array}$} & \multicolumn{3}{|c|}{ Coordinates (Angstroms) } \\
\hline & & $\mathrm{X}$ & Y & $\mathrm{z}$ \\
\hline 1 & 6 & -0.447095 & -0.123318 & 0.043146 \\
\hline 2 & 6 & 0.287108 & -1.160091 & 0.786747 \\
\hline 3 & 1 & 1.225754 & -0.907224 & 1.269635 \\
\hline 4 & 1 & -0.301833 & -1.963027 & 1.221950 \\
\hline 5 & 7 & 0.353207 & -1.049682 & -0.703308 \\
\hline 6 & 1 & -0.219913 & -1.746747 & -1.183252 \\
\hline 7 & 1 & 4.185647 & -0.723167 & -0.283164 \\
\hline 8 & 7 & 3.326802 & -0.254061 & -0.002754 \\
\hline 9 & 1 & 3.496963 & 0.748170 & -0.054797 \\
\hline 10 & 1 & 2.605449 & -0.478457 & -0.689008 \\
\hline 11 & 17 & -2.199061 & -0.254557 & -0.059464 \\
\hline 12 & 17 & 0.093637 & 1.542622 & 0.040741 \\
\hline
\end{tabular}

2,2-Dichloroaziridine in THF: $E=-1051.8341015$; Thermal Energy Correction $=0.056802$.

\begin{tabular}{|c|c|c|c|c|}
\hline Number & Atomic & x & $\begin{array}{l}\text { (Angst } \\
Y\end{array}$ & mS) \\
\hline 1 & 6 & -0.001011 & 0.227465 & -0.004080 \\
\hline 2 & 6 & -0.114312 & 1.533217 & -0.673134 \\
\hline 3 & 7 & -0.047303 & 1.398406 & 0.812601 \\
\hline 4 & 1 & -0.964154 & 1.528407 & 1.268110 \\
\hline 5 & 1 & 0.767190 & 1.979827 & -1.123262 \\
\hline 6 & 1 & -1.071044 & 1.777966 & -1.128336 \\
\hline 7 & 17 & 1.520677 & -0.650824 & -0.004163 \\
\hline 8 & 17 & -1.385908 & -0.857360 & -0.033569 \\
\hline
\end{tabular}

2,2-Dichloroaziridine in $\mathrm{MeCN}: \mathrm{E}=-1051.8360614$; Thermal Energy Correction $=0.056583$.

\begin{tabular}{|c|c|c|c|c|}
\hline Center & Atomic & Coorc & nates (Angs & coms ) \\
\hline Number & Number & $\mathrm{X}$ & $\mathrm{Y}$ & Z \\
\hline 1 & 6 & -0.003244 & 0.228109 & -0.004529 \\
\hline 2 & 6 & -0.111578 & 1.533900 & -0.673833 \\
\hline 3 & 1 & 0.771263 & 1.976695 & -1.125579 \\
\hline 4 & 1 & -1.068982 & 1.783589 & -1.125602 \\
\hline 5 & 7 & -0.046343 & 1.398432 & 0.813591 \\
\hline 6 & 1 & -0.968539 & 1.532349 & 1.266255 \\
\hline 7 & 17 & 1.520227 & -0.651882 & -0.003707 \\
\hline 8 & 17 & -1.386133 & -0.857160 & -0.033942 \\
\hline
\end{tabular}


2,2-Dichloroaziridine in $\mathrm{H}_{2} \mathrm{O}: \mathrm{E}=-1051.8364581$; Thermal Energy Correction $=0.056535$.

\begin{tabular}{ccrrr} 
Center & Atomic & \multicolumn{3}{c}{ Coordinates } \\
Number & Number & X Angtroms) & Y & $Z$ \\
-1 & 6 & 0.110739 & 1.534265 & -0.673527 \\
1 & 6 & 0.003066 & 0.228727 & -0.004347 \\
2 & 1 & -0.773096 & 1.975909 & -1.124786 \\
3 & 1 & 1.067306 & 1.785119 & -1.126441 \\
4 & 17 & 1.386582 & -0.856949 & -0.034040 \\
5 & 17 & -1.520018 & -0.652580 & -0.003664 \\
6 & 7 & 0.045963 & 1.398680 & 0.813543 \\
7 & 1 & 0.969626 & 1.532243 & 1.264639 \\
8 & & & &
\end{tabular}

2-Bromoaziridine $+\mathrm{NH}_{3}$ Transition State (No Solvent): $\mathrm{E}=-649.0984237$; Thermal Energy Correction $=0.105752$. Unique imaginary frequency $=-669.4331 \mathrm{~cm}^{-1}$.

\begin{tabular}{cccrr} 
Center & Atomic & \multicolumn{3}{c}{ Coordinates (Angstroms) } \\
Number & Number & X & \multicolumn{1}{c}{$Y$} & \multicolumn{1}{c}{ Z } \\
--1 & 1 & -2.714193 & 0.432026 & 0.280485 \\
1 & 6 & -1.742449 & 0.304336 & -0.183996 \\
2 & 1 & -1.718726 & 0.264882 & -1.266801 \\
3 & 6 & -0.601601 & 0.899947 & 0.473809 \\
4 & 7 & -1.693595 & -1.724164 & 0.018968 \\
5 & 7 & -0.769487 & 2.140833 & -0.058977 \\
6 & 1 & -0.561933 & 0.872978 & 1.561052 \\
7 & 1 & -2.158939 & -2.262982 & -0.712717 \\
8 & 1 & -2.012358 & -2.062891 & 0.927229 \\
9 & 1 & -0.679422 & -1.856657 & -0.049941 \\
10 & 1 & -0.717619 & 2.112783 & -1.085674 \\
11 & 35 & 1.196259 & -0.218358 & -0.031784 \\
12 & & & &
\end{tabular}

2-Bromoaziridine $+\mathrm{NH}_{3}$ Transition State in THF: $\mathrm{E}=-2762.3167609$; Thermal Energy Correction $=0.103927$. Unique imaginary frequency $=-651.1547 \mathrm{~cm}^{-1}$.

\begin{tabular}{cccrr}
$\begin{array}{c}\text { Center } \\
\text { Number }\end{array}$ & $\begin{array}{c}\text { Atomic } \\
\text { Number }\end{array}$ & \multicolumn{3}{c}{ Coordinates (Angstroms) } \\
- X & Y & \multicolumn{1}{c}{$Z$} \\
\hline 1 & 1 & -2.610053 & 0.736029 & 0.271758 \\
2 & 6 & -1.662591 & 0.517048 & -0.205883 \\
3 & 1 & -1.637356 & 0.444814 & -1.285963 \\
4 & 6 & -0.456645 & 0.894776 & 0.483252 \\
5 & 7 & -1.968860 & -1.557084 & 0.014770 \\
6 & 7 & -0.537805 & 2.166395 & -0.041085 \\
7 & 1 & -0.450964 & 0.845191 & 1.572992 \\
8 & 1 & -2.946373 & -1.853884 & -0.067337 \\
9 & 1 & -1.636071 & -1.822812 & 0.945869 \\
10 & 1 & -1.417788 & -2.081314 & -0.669968 \\
11 & 1 & -0.401476 & 2.159216 & -1.065736 \\
12 & 35 & 1.181776 & -0.318953 & -0.033761
\end{tabular}


2-Bromoaziridine $+\mathrm{NH}_{3}$ Transition State in $\mathrm{MeCN}: \mathrm{E}=-2762.3211698$; Thermal Energy Correction $=0.103536$. Unique imaginary frequency $=-666.3682 \mathrm{~cm}^{-1}$.

\begin{tabular}{|c|c|c|c|c|c|}
\hline Center & Atomic & & Coord: & (Angst & oms) \\
\hline Number & Number & & $\mathrm{x}$ & Y & Z \\
\hline 1 & 1 & 0 & -2.589466 & 0.787226 & 0.264656 \\
\hline 2 & 6 & 0 & -1.645742 & 0.551811 & -0.211842 \\
\hline 3 & 1 & 0 & -1.616651 & 0.470258 & -1.291081 \\
\hline 4 & 6 & 0 & -0.433503 & 0.887958 & 0.486753 \\
\hline 5 & 7 & 0 & -2.020971 & -1.522932 & 0.014399 \\
\hline 6 & 7 & 0 & -0.499009 & 2.165111 & -0.037053 \\
\hline 7 & 1 & 0 & -0.435697 & 0.832277 & 1.576781 \\
\hline 8 & 1 & 0 & -3.013166 & -1.772982 & -0.054120 \\
\hline 9 & 1 & 0 & -1.691663 & -1.800863 & 0.943895 \\
\hline 10 & 1 & 0 & -1.506425 & -2.078651 & -0.674946 \\
\hline 11 & 1 & 0 & -0.341042 & 2.159652 & -1.060449 \\
\hline 12 & 35 & 0 & 1.180270 & -0.335165 & -0.034160 \\
\hline
\end{tabular}

2-Bromoaziridine $+\mathrm{NH}_{3}$ Transition State in $\mathrm{H}_{2} \mathrm{O}: \quad \mathrm{E}=-2762.3219111$; Thermal Energy Correction $=0.103442$. Unique imaginary frequency $=-670.6101 \mathrm{~cm}^{-1}$.

\begin{tabular}{|c|c|c|c|c|}
\hline Center & Atomic & Coorc & nates (Angs & coms ) \\
\hline Number & Number & $\mathrm{X}$ & Y & Z \\
\hline 1 & 1 & -2.586548 & 0.795706 & 0.256940 \\
\hline 2 & 6 & -1.642347 & 0.556939 & -0.216787 \\
\hline 3 & 1 & -1.609524 & 0.474295 & -1.295863 \\
\hline 4 & 6 & -0.431215 & 0.885110 & 0.487345 \\
\hline 5 & 7 & -2.030559 & -1.516011 & 0.015123 \\
\hline 6 & 7 & -0.492515 & 2.164227 & -0.033619 \\
\hline 7 & 1 & -0.436992 & 0.825271 & 1.577232 \\
\hline 8 & 1 & -3.024990 & -1.759148 & -0.047059 \\
\hline 9 & 1 & -1.698998 & -1.789850 & 0.945211 \\
\hline 10 & 1 & -1.523101 & -2.080132 & -0.672868 \\
\hline 11 & 1 & -0.329371 & 2.159255 & -1.056583 \\
\hline 12 & 35 & 1.180355 & -0.337577 & -0.034311 \\
\hline
\end{tabular}

2-Bromoaziridine $+\mathrm{NH}_{3}$ Dipolar Complex (No Solvent): $\mathrm{E}=-649.1644480$; Thermal Energy Correction $=0.107826$.

\begin{tabular}{|c|c|c|c|c|}
\hline $\begin{array}{l}\text { Center } \\
\text { Number }\end{array}$ & $\begin{array}{l}\text { Atomic } \\
\text { Number }\end{array}$ & \multicolumn{3}{|c|}{ Coordinates (Angstroms) } \\
\hline 1 & 6 & -0.336920 & 1.937237 & 0.624291 \\
\hline 2 & 6 & 0.290232 & 1.144418 & -0.449957 \\
\hline 3 & 1 & -0.423739 & 1.460585 & 1.597513 \\
\hline 4 & 1 & -0.247532 & 3.019404 & 0.634067 \\
\hline 5 & 7 & -1.117436 & 1.376714 & -0.506100 \\
\hline 6 & 1 & -1.689566 & 0.561114 & -0.241259 \\
\hline 7 & 1 & -1.674060 & -1.783160 & 0.055135 \\
\hline 8 & 7 & -2.544787 & -1.254393 & 0.055805 \\
\hline 9 & 1 & -3.062851 & -1.514380 & 0.893287 \\
\hline 10 & 1 & -3.094951 & -1.560532 & -0.744790 \\
\hline 11 & 35 & 1.005691 & -0.602875 & 0.032654 \\
\hline 12 & 1 & 0.909190 & 1.571403 & -1.230785 \\
\hline
\end{tabular}


2-Bromoaziridine in THF: $E=-2704.8724235$; Thermal Energy Correction $=0.065234$.

\begin{tabular}{|c|c|c|c|c|}
\hline \multirow{2}{*}{$\begin{array}{l}\text { Center } \\
\text { Number }\end{array}$} & \multirow{2}{*}{$\begin{array}{l}\text { Atomic } \\
\text { Number }\end{array}$} & \multicolumn{3}{|c|}{ Coordinates (Angstroms) } \\
\hline & & $\mathrm{x}$ & $\mathrm{Y}$ & $\mathrm{Z}$ \\
\hline 1 & 6 & -0.818938 & -0.036224 & 0.593564 \\
\hline 2 & 6 & -1.843221 & 0.695453 & -0.171518 \\
\hline 3 & 1 & -0.834522 & -0.116471 & 1.677095 \\
\hline 4 & 1 & -1.525646 & 1.189002 & -1.086261 \\
\hline 5 & 1 & -2.684504 & 1.146885 & 0.346065 \\
\hline 6 & 7 & -1.807846 & -0.794230 & -0.136556 \\
\hline 7 & 1 & -1.436889 & -1.178300 & -1.018047 \\
\hline 8 & 35 & 1.003127 & 0.016089 & -0.042721 \\
\hline
\end{tabular}

2-Bromoaziridine in $\mathrm{MeCN}$ : $\mathrm{E}=-2704.9231979$; Thermal Energy Correction $=0.065015$.

\begin{tabular}{|c|c|c|c|c|}
\hline Center & Atomic & Coors & hates & coms) \\
\hline Number & Number & $\mathrm{X}$ & $\mathrm{Y}$ & Z \\
\hline 1 & 6 & -0.819485 & -0.033075 & 0.593317 \\
\hline 2 & 6 & -1.843922 & 0.694985 & -0.174059 \\
\hline 3 & 1 & -0.833677 & -0.106179 & 1.678097 \\
\hline 4 & 1 & -1.529469 & 1.181130 & -1.093927 \\
\hline 5 & 1 & -2.682432 & 1.150776 & 0.344529 \\
\hline 6 & 7 & -1.808672 & -0.795891 & -0.132828 \\
\hline 7 & 1 & -1.437898 & -1.180796 & -1.017149 \\
\hline 8 & 35 & 1.003561 & 0.015853 & -0.042780 \\
\hline
\end{tabular}

2-Bromoaziridine in $\mathrm{H}_{2} \mathrm{O}: \mathrm{E}=-2704.9235649$; Thermal Energy Correction $=0.064961$.

\begin{tabular}{|c|c|c|c|c|}
\hline $\begin{array}{l}\text { Center } \\
\text { Number }\end{array}$ & $\begin{array}{l}\text { Atomic } \\
\text { Number }\end{array}$ & \multicolumn{3}{|c|}{$\begin{array}{cc}\text { Coordinates (Angstroms) } \\
\mathrm{X}\end{array}$} \\
\hline 1 & 6 & 0.819669 & 0.032078 & 0.593339 \\
\hline 2 & 6 & 1.844340 & -0.694573 & -0.174779 \\
\hline 3 & 1 & 0.833463 & 0.103269 & 1.678328 \\
\hline 4 & 1 & 1.531049 & -1.179309 & -1.095802 \\
\hline 5 & 1 & 2.682754 & -1.150498 & 0.343952 \\
\hline 6 & 7 & 1.808424 & 0.796586 & -0.132072 \\
\hline 7 & 1 & 1.434655 & 1.180410 & -1.016395 \\
\hline 8 & 35 & -1.003570 & -0.015857 & -0.042770 \\
\hline
\end{tabular}


2,2-Dibromoaziridine $+\mathrm{NH}_{3}$ Transition State (No Solvent): $\mathrm{E}=-5334.6030238$; Thermal Energy Correction $=0.095820$. Unique imaginary frequency $=-649.6339 \mathrm{~cm}^{-1}$.

\begin{tabular}{|c|c|c|c|c|}
\hline \multirow{2}{*}{$\begin{array}{l}\text { Center } \\
\text { Number }\end{array}$} & \multirow{2}{*}{$\begin{array}{l}\text { Atomic } \\
\text { Number }\end{array}$} & \multicolumn{3}{|c|}{ Coordinates (Angstroms) } \\
\hline & & $\mathrm{X}$ & $\mathrm{Y}$ & $\mathrm{z}$ \\
\hline 1 & 1 & -0.793482 & 2.008549 & 1.412154 \\
\hline 2 & 6 & 0.069864 & 1.588368 & 0.914227 \\
\hline 3 & 1 & 1.022443 & 2.099080 & 0.982242 \\
\hline 4 & 6 & 0.100699 & 0.146366 & 0.766990 \\
\hline 5 & 7 & -0.394569 & 2.329962 & -0.919181 \\
\hline 6 & 7 & 0.164783 & 0.030217 & 2.101234 \\
\hline 7 & 1 & -0.729563 & 3.293446 & -0.968417 \\
\hline 8 & 1 & -1.139276 & 1.694561 & -1.217157 \\
\hline 9 & 1 & 0.393868 & 2.219741 & -1.558274 \\
\hline 10 & 1 & 1.014167 & -0.427433 & 2.444065 \\
\hline 11 & 35 & -1.592756 & -0.624195 & -0.194037 \\
\hline 12 & 35 & 1.616098 & -0.456308 & -0.361857 \\
\hline
\end{tabular}

2,2-Dibromoaziridine $+\mathrm{NH}_{3}$ Transition State in THF: $\mathrm{E}=-5334.6207664$; Thermal Energy Correction $=0.094374$. Unique imaginary frequency $=-666.1808 \mathrm{~cm}^{-1}$.

\begin{tabular}{|c|c|c|c|c|}
\hline Center & Atomic & Coorc & nates & roms ) \\
\hline Number & Number & X & $\mathrm{Y}$ & Z \\
\hline 1 & 1 & -0.852601 & 1.956243 & 1.424123 \\
\hline 2 & 6 & 0.038451 & 1.542044 & 0.973202 \\
\hline 3 & 1 & 0.983310 & 2.064420 & 1.065090 \\
\hline 4 & 6 & 0.081801 & 0.116971 & 0.749686 \\
\hline 5 & 7 & -0.378275 & 2.421415 & -0.876990 \\
\hline 6 & 7 & 0.137253 & -0.023551 & 2.092802 \\
\hline 7 & 1 & -0.045319 & 1.810805 & -1.628396 \\
\hline 8 & 1 & 0.062664 & 3.338880 & -1.000121 \\
\hline 9 & 1 & -1.388114 & 2.538596 & -1.002254 \\
\hline 10 & 1 & 1.040901 & -0.390623 & 2.435075 \\
\hline 11 & 35 & 1.614452 & -0.453415 & -0.362484 \\
\hline 12 & 35 & -1.581172 & -0.633941 & -0.212988 \\
\hline
\end{tabular}

2,2-Dibromoaziridine $+\mathrm{NH}_{3}$ Transition State in MeCN: $\mathrm{E}=-5334.6247896$; Thermal Energy Correction $=0.093950$. Unique imaginary frequency $=-669.5757 \mathrm{~cm}^{-1}$.

\begin{tabular}{|c|c|c|c|c|}
\hline \multirow{2}{*}{$\begin{array}{l}\text { Center } \\
\text { Number }\end{array}$} & \multirow{2}{*}{$\begin{array}{l}\text { Atomic } \\
\text { Number }\end{array}$} & \multicolumn{3}{|c|}{ Coordinates (Angstroms) } \\
\hline & & $\mathrm{X}$ & $\mathrm{Y}$ & $\mathrm{Z}$ \\
\hline 1 & 1 & -0.865771 & 1.948493 & 1.416812 \\
\hline 2 & 6 & 0.033631 & 1.532718 & 0.984139 \\
\hline 3 & 1 & 0.977824 & 2.053830 & 1.090875 \\
\hline 4 & 6 & 0.077624 & 0.111413 & 0.744476 \\
\hline 5 & 7 & -0.353377 & 2.442720 & -0.869828 \\
\hline 6 & 7 & 0.125053 & -0.035496 & 2.090011 \\
\hline 7 & 1 & -0.028720 & 1.845542 & -1.636416 \\
\hline 8 & 1 & 0.109382 & 3.353408 & -0.966986 \\
\hline 9 & 1 & -1.359786 & 2.588410 & -0.999527 \\
\hline 10 & 1 & 1.041317 & -0.376058 & 2.434656 \\
\hline 11 & 35 & 1.612809 & -0.459185 & -0.359925 \\
\hline 12 & 35 & -1.582623 & -0.630214 & -0.218715 \\
\hline
\end{tabular}


2,2-Dibromoaziridine $+\mathrm{NH}_{3}$ Transition State in $\mathrm{H}_{2} \mathrm{O}: \quad \mathrm{E}=-5334.6254642$; Thermal Energy Correction $=0.093850$. Unique imaginary frequency $=-669.8509 \mathrm{~cm}^{-1}$.

\begin{tabular}{|c|c|c|c|c|}
\hline \multirow{2}{*}{$\begin{array}{l}\text { Center } \\
\text { Number }\end{array}$} & Atomic & \multicolumn{3}{|c|}{ Coordinates (Angstroms) } \\
\hline & Number & $\mathrm{X}$ & Y & Z \\
\hline 1 & 1 & -0.868135 & 1.947140 & 1.413470 \\
\hline 2 & 6 & 0.034101 & 1.528853 & 0.988994 \\
\hline 3 & 1 & 0.979385 & 2.045487 & 1.107171 \\
\hline 4 & 6 & 0.074623 & 0.108476 & 0.744442 \\
\hline 5 & 7 & -0.326646 & 2.447240 & -0.866809 \\
\hline 6 & 7 & 0.115973 & -0.043771 & 2.090496 \\
\hline 7 & 1 & -0.011140 & 1.842088 & -1.631227 \\
\hline 8 & 1 & 0.157961 & 3.346893 & -0.961719 \\
\hline 9 & 1 & -1.328570 & 2.616653 & -1.003132 \\
\hline 10 & 1 & 1.034922 & -0.377545 & 2.436320 \\
\hline 11 & 35 & 1.610250 & -0.465329 & -0.357825 \\
\hline 12 & 35 & -1.585737 & -0.622356 & -0.222955 \\
\hline
\end{tabular}

2,2-Dibromoaziridine $+\mathrm{NH}_{3}$ Dipolar Complex (No Solvent): $\mathrm{E}=-5334.6562539$; Thermal Energy Correction $=0.097932$.

\begin{tabular}{|c|c|c|c|c|}
\hline Center & Atomic & Coors & nates & roms) \\
\hline Number & Number & X & $\mathrm{Y}$ & Z \\
\hline 1 & 6 & -0.134862 & 1.197167 & 1.504973 \\
\hline 2 & 6 & 0.162932 & 0.384589 & 0.314517 \\
\hline 3 & 1 & 0.660544 & 1.761091 & 1.982680 \\
\hline 4 & 1 & -0.943806 & 0.854419 & 2.146368 \\
\hline 5 & 7 & -0.456395 & 1.633178 & 0.115838 \\
\hline 6 & 1 & -1.458527 & 1.582583 & -0.138113 \\
\hline 7 & 35 & -0.857149 & -1.255092 & 0.120312 \\
\hline 8 & 35 & 1.960361 & 0.181989 & -0.329636 \\
\hline 9 & 7 & -3.289439 & 1.278712 & -0.790834 \\
\hline 10 & 1 & -4.108018 & 1.532827 & -0.240128 \\
\hline 11 & 1 & -3.297470 & 0.265777 & -0.900727 \\
\hline 12 & 1 & -3.412714 & 1.688116 & -1.715711 \\
\hline
\end{tabular}

2,2-Dibromoaziridine in THF: $E=-5278.2172829$; Thermal Energy Correction $=0.055842$.

\begin{tabular}{|c|c|c|c|c|}
\hline Center & Atomic & Coorc & nates & roms ) \\
\hline Number & Number & X & $\mathrm{Y}$ & Z \\
\hline 1 & 6 & 0.041422 & 1.882639 & -0.683385 \\
\hline 2 & 6 & 0.000350 & 0.584706 & 0.007818 \\
\hline 3 & 1 & -0.863337 & 2.267939 & -1.144622 \\
\hline 4 & 1 & 0.986012 & 2.178365 & -1.134514 \\
\hline 5 & 7 & -0.029374 & 1.761966 & 0.808216 \\
\hline 6 & 1 & 0.871626 & 1.956844 & 1.274738 \\
\hline 7 & 35 & 1.584090 & -0.501496 & -0.014406 \\
\hline 8 & 35 & -1.613785 & -0.456817 & -0.002728 \\
\hline
\end{tabular}


2,2-Dibromoaziridine in $\mathrm{MeCN}: \mathrm{E}=-5278.2193043$; Thermal Energy Correction $=0.055624$.

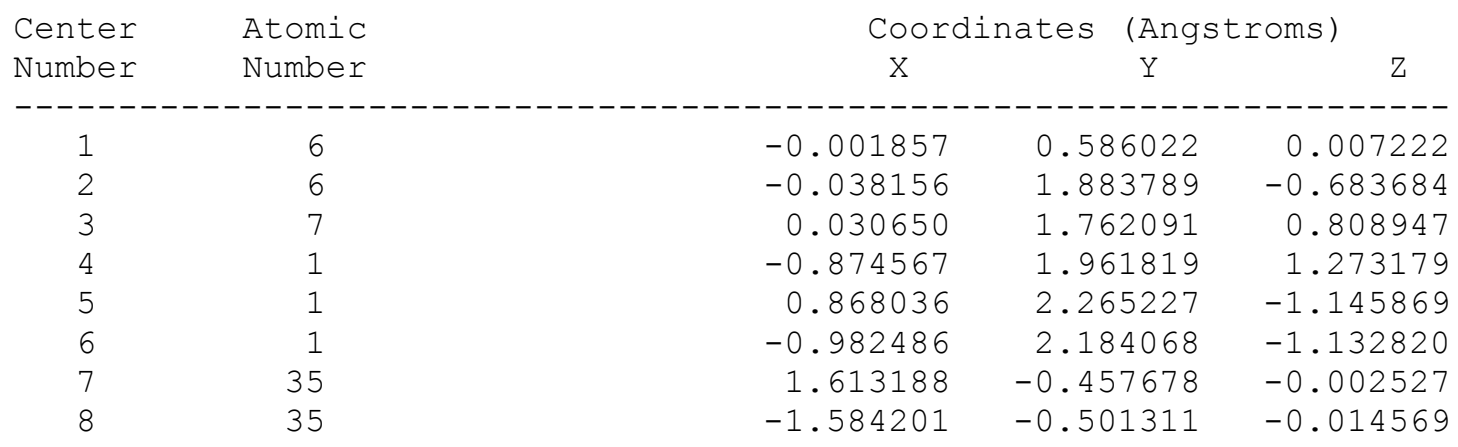

2,2-Dibromoaziridine in $\mathrm{H}_{2} \mathrm{O}: \quad \mathrm{E}=-5278.2196994$; Thermal Energy Correction $=0.055573$.

\begin{tabular}{|c|c|c|c|c|}
\hline Center & Atomic & Coors & ates & roms) \\
\hline Number & Number & $\mathrm{x}$ & $\mathrm{Y}$ & Z \\
\hline 1 & 6 & 0.038762 & 1.884224 & -0.683637 \\
\hline 2 & 6 & 0.002183 & 0.586451 & 0.007107 \\
\hline 3 & 1 & -0.867116 & 2.265780 & -1.146536 \\
\hline 4 & 1 & 0.983537 & 2.184708 & -1.131774 \\
\hline 5 & 7 & -0.030712 & 1.762071 & 0.809206 \\
\hline 6 & 1 & 0.875677 & 1.960911 & 1.272914 \\
\hline 7 & 35 & 1.584028 & -0.501543 & -0.014571 \\
\hline 8 & 35 & -1.613251 & -0.457599 & -0.002568 \\
\hline
\end{tabular}

Chloromethylethylamine $+\mathrm{NH}_{3}$ Transition State (Attack on Ethyl Group) (No Solvent): $\mathrm{E}=-689.5168635$; Thermal Energy Correction $=0.161631$. Unique imaginary frequency $=-642.3631 \mathrm{~cm}^{-1}$.

\begin{tabular}{|c|c|c|c|c|c|}
\hline \multirow{2}{*}{$\begin{array}{l}\text { Center } \\
\text { Number }\end{array}$} & \multirow{2}{*}{$\begin{array}{l}\text { Atomic } \\
\text { Number }\end{array}$} & \multirow{2}{*}{$\begin{array}{c}\text { Atomic } \\
\text { Type }\end{array}$} & \multicolumn{3}{|c|}{ Coordinates (Angstroms) } \\
\hline & & & $\mathrm{X}$ & $Y$ & Z \\
\hline 1 & 1 & 0 & -0.040325 & 0.031080 & -0.308654 \\
\hline 2 & 6 & 0 & 0.662585 & 0.849068 & -0.339565 \\
\hline 3 & 6 & 0 & 1.086135 & 1.562539 & 0.919215 \\
\hline 4 & 1 & 0 & 1.008619 & 1.206563 & -1.300252 \\
\hline 5 & 7 & 0 & -1.136731 & 1.865520 & -0.545200 \\
\hline 6 & 7 & 0 & 2.023188 & -0.616177 & -0.328635 \\
\hline 7 & 1 & 0 & -1.344592 & 2.686219 & 0.024179 \\
\hline 8 & 1 & 0 & -1.721160 & 1.071169 & -0.228199 \\
\hline 9 & 1 & 0 & -1.402274 & 2.073753 & -1.508587 \\
\hline 10 & 1 & 0 & 0.436852 & 1.265500 & 1.746360 \\
\hline 11 & 1 & 0 & 2.115198 & 1.318400 & 1.190959 \\
\hline 12 & 1 & 0 & 3.039381 & -0.544583 & -0.454660 \\
\hline 13 & 6 & 0 & 1.575958 & -1.805456 & -0.132799 \\
\hline 14 & 1 & 0 & 2.234958 & -2.674267 & -0.101989 \\
\hline 15 & 1 & 0 & 0.500069 & -1.935623 & 0.009556 \\
\hline 16 & 17 & 0 & -1.882959 & -1.148553 & 0.210432 \\
\hline 17 & 1 & 0 & 1.030322 & 2.644896 & 0.789684 \\
\hline
\end{tabular}




\begin{tabular}{|c|c|c|c|c|c|}
\hline \multirow{2}{*}{$\begin{array}{l}\text { Center } \\
\text { Number }\end{array}$} & \multirow{2}{*}{$\begin{array}{l}\text { Atomic } \\
\text { Number }\end{array}$} & \multirow{2}{*}{$\begin{array}{l}\text { Atomic } \\
\text { Type }\end{array}$} & \multicolumn{3}{|c|}{ Coordinates (Angstroms) } \\
\hline & & & $\mathrm{X}$ & Y & Z \\
\hline 1 & 6 & 0 & 2.924526 & 0.012813 & -0.400885 \\
\hline 2 & 1 & 0 & 2.858324 & 1.079601 & -0.165420 \\
\hline 3 & 1 & 0 & 2.824875 & -0.108275 & -1.482453 \\
\hline 4 & 1 & 0 & 3.913755 & -0.341053 & -0.094353 \\
\hline 5 & 6 & 0 & 1.829114 & -0.761913 & 0.312824 \\
\hline 6 & 1 & 0 & 1.953322 & -0.658872 & 1.406417 \\
\hline 7 & 6 & 0 & -0.552919 & -0.983555 & 0.515556 \\
\hline 8 & 1 & 0 & -0.693897 & -0.711080 & 1.571147 \\
\hline 9 & 1 & 0 & -0.396366 & -2.061277 & 0.440201 \\
\hline 10 & 7 & 0 & 0.520085 & -0.300391 & -0.147459 \\
\hline 11 & 1 & 0 & 0.424264 & 0.708734 & 0.010526 \\
\hline 12 & 7 & 0 & -0.484495 & 2.613414 & 0.176673 \\
\hline 13 & 1 & 0 & -0.566403 & 3.284078 & 0.938832 \\
\hline 14 & 1 & 0 & -1.409061 & 2.214462 & 0.017078 \\
\hline 15 & 1 & 0 & -0.235340 & 3.137956 & -0.660147 \\
\hline 16 & 1 & 0 & 1.911388 & -1.830205 & 0.079053 \\
\hline 17 & 17 & 0 & -2.119901 & -0.618194 & -0.284139 \\
\hline
\end{tabular}

\begin{tabular}{|c|c|c|c|c|c|}
\hline \multirow{2}{*}{$\begin{array}{l}\text { Center } \\
\text { Number }\end{array}$} & \multirow{2}{*}{$\begin{array}{l}\text { Atomic } \\
\text { Number }\end{array}$} & \multirow{2}{*}{$\begin{array}{c}\text { Atomic } \\
\text { Type }\end{array}$} & \multicolumn{3}{|c|}{ Coordinates (Angstroms) } \\
\hline & & & $\mathrm{x}$ & $\mathrm{Y}$ & Z \\
\hline 1 & 1 & 0 & -0.835548 & -0.001806 & 0.497177 \\
\hline 2 & 6 & 0 & 0.221648 & 0.217002 & 0.577982 \\
\hline 3 & 6 & 0 & 1.060706 & 0.121518 & -0.671463 \\
\hline 4 & 1 & 0 & 0.691249 & 0.394773 & 1.534823 \\
\hline 5 & 7 & 0 & 0.253782 & -1.816300 & 0.911568 \\
\hline 6 & 7 & 0 & -0.308474 & 2.102402 & 0.427524 \\
\hline 7 & 1 & 0 & 1.005581 & -2.402139 & 0.546577 \\
\hline 8 & 1 & 0 & -0.633474 & -2.018716 & 0.420935 \\
\hline 9 & 1 & 0 & 0.121127 & -2.053134 & 1.895622 \\
\hline 10 & 1 & 0 & 0.703912 & -0.700634 & -1.291664 \\
\hline 11 & 1 & 0 & 1.008126 & 1.035091 & -1.260304 \\
\hline 12 & 1 & 0 & 0.262103 & 2.929510 & 0.636516 \\
\hline 13 & 6 & 0 & -1.525788 & 2.330832 & 0.083281 \\
\hline 14 & 1 & c & -1.925296 & 3.342889 & 0.011049 \\
\hline 15 & 1 & 0 & -2.164057 & 1.473658 & -0.147635 \\
\hline 16 & 17 & c & 2.785670 & -0.154257 & -0.298045 \\
\hline 17 & 17 & 0 & -2.573334 & -1.023291 & -0.416987 \\
\hline
\end{tabular}


2-Chloroethylchloromethylamine $+\mathrm{NH}_{3}$ Ground State (No Solvent): $\mathrm{E}=$ 1148.7117874; Thermal Energy Correction $=0.155306$.

\begin{tabular}{|c|c|c|c|c|c|}
\hline \multirow{2}{*}{$\begin{array}{l}\text { Center } \\
\text { Number }\end{array}$} & \multirow{2}{*}{$\begin{array}{l}\text { Atomic } \\
\text { Number }\end{array}$} & \multirow{2}{*}{$\begin{array}{c}\text { Atomic } \\
\text { Type }\end{array}$} & \multicolumn{3}{|c|}{ Coordinates (Angstroms) } \\
\hline & & & $\mathrm{x}$ & $\mathrm{Y}$ & $\mathrm{Z}$ \\
\hline 1 & 1 & 0 & 1.647534 & 1.107312 & -0.451198 \\
\hline 2 & 6 & 0 & 1.750025 & 0.025965 & -0.537341 \\
\hline 3 & 1 & 0 & 1.705853 & -0.275775 & -1.586554 \\
\hline 4 & 6 & 0 & 0.667767 & -0.662107 & 0.273738 \\
\hline 5 & 1 & 0 & 0.849122 & -1.748034 & 0.307223 \\
\hline 6 & 1 & 0 & 0.699040 & -0.286886 & 1.301881 \\
\hline 7 & 6 & 0 & -1.724041 & -0.836880 & 0.511934 \\
\hline 8 & 1 & 0 & -1.637081 & -0.423032 & 1.517997 \\
\hline 9 & 1 & 0 & -1.757882 & -1.932242 & 0.572376 \\
\hline 10 & 17 & 0 & -3.286578 & -0.316365 & -0.166210 \\
\hline 11 & 17 & 0 & 3.360731 & -0.437063 & 0.079168 \\
\hline 12 & 7 & 0 & -0.640797 & -0.317713 & -0.295178 \\
\hline 13 & 1 & 0 & -0.717749 & -0.699680 & -1.240497 \\
\hline 14 & 1 & 0 & -0.573103 & 1.936979 & -0.056739 \\
\hline 15 & 7 & 0 & -0.064533 & 2.791834 & 0.178278 \\
\hline 16 & 1 & 0 & -0.482158 & 3.188579 & 1.017959 \\
\hline 17 & 1 & 0 & -0.219371 & 3.460339 & -0.574402 \\
\hline
\end{tabular}

Fluoromethylethylamine $+\mathrm{NH}_{3}$ Transition State (Attack on Ethyl Group) (No Solvent): $E=-329.489728$; Thermal Energy Correction $=0.160083$. Unique imaginary frequency $=-645.3696 \mathrm{~cm}^{-1}$.

\begin{tabular}{|c|c|c|c|c|c|}
\hline \multirow{2}{*}{$\begin{array}{l}\text { Center } \\
\text { Number }\end{array}$} & \multirow{2}{*}{$\begin{array}{l}\text { Atomic } \\
\text { Number }\end{array}$} & \multirow{2}{*}{$\begin{array}{l}\text { Atomic } \\
\text { Type }\end{array}$} & \multicolumn{3}{|c|}{ Coordinates (Angstroms) } \\
\hline & & & $\mathrm{x}$ & $\mathrm{Y}$ & Z \\
\hline 1 & 1 & 0 & 0.135913 & 0.368913 & 0.107274 \\
\hline 2 & 6 & 0 & 0.241871 & -0.626735 & -0.306062 \\
\hline 3 & 6 & 0 & 0.484127 & -1.852870 & 0.530880 \\
\hline 4 & 1 & 0 & 0.255213 & -0.725226 & -1.383865 \\
\hline 5 & 7 & 0 & 2.186317 & 0.170342 & -0.340369 \\
\hline 6 & 7 & 0 & -1.764010 & -0.556516 & -0.279240 \\
\hline 7 & 1 & 0 & 2.846175 & -0.119421 & 0.381527 \\
\hline 8 & 1 & 0 & 1.790534 & 1.109215 & -0.088900 \\
\hline 9 & 1 & 0 & 2.692205 & 0.230481 & -1.224027 \\
\hline 10 & 1 & 0 & 0.564905 & -1.586289 & 1.587097 \\
\hline 11 & 1 & 0 & -0.329779 & -2.573565 & 0.418227 \\
\hline 12 & 1 & 0 & -2.479968 & -1.271765 & -0.452274 \\
\hline 13 & 6 & 0 & -2.212829 & 0.619812 & -0.010955 \\
\hline 14 & 1 & 0 & -3.282951 & 0.829933 & 0.031651 \\
\hline 15 & 1 & 0 & -1.480446 & 1.416170 & 0.178014 \\
\hline 16 & 1 & 0 & 1.404022 & -2.351826 & 0.220707 \\
\hline 17 & 9 & 0 & 0.427668 & 2.059484 & 0.364294 \\
\hline
\end{tabular}

Fluoromethylethylamine $+\mathrm{NH}_{3}$ Ground State (No Solvent): $\mathrm{E}=$ 329.6059204; Thermal Energy Correction $=0.164284$. 


\begin{tabular}{|c|c|c|c|c|c|}
\hline \multirow{2}{*}{$\begin{array}{l}\text { Center } \\
\text { Number }\end{array}$} & \multirow{2}{*}{$\begin{array}{l}\text { Atomic } \\
\text { Number }\end{array}$} & \multirow{2}{*}{$\begin{array}{l}\text { Atomic } \\
\text { Type }\end{array}$} & \multicolumn{3}{|c|}{ Coordinates (Angstroms) } \\
\hline & & & $\mathrm{X}$ & $\mathrm{Y}$ & Z \\
\hline 1 & 6 & 0 & 1.513761 & -1.157475 & 0.751039 \\
\hline 2 & 1 & 0 & 0.777093 & -1.962603 & 0.662467 \\
\hline 3 & 1 & 0 & 1.224418 & -0.520255 & 1.588816 \\
\hline 4 & 1 & 0 & 2.485564 & -1.613470 & 0.971800 \\
\hline 5 & 6 & 0 & 1.588064 & -0.357755 & -0.548518 \\
\hline 6 & 1 & 0 & 1.928773 & -1.006603 & -1.362960 \\
\hline 7 & 6 & 0 & -0.082018 & 1.418881 & -0.343782 \\
\hline 8 & 1 & 0 & -0.970788 & 1.831682 & -0.820669 \\
\hline 9 & 1 & 0 & 0.723507 & 2.151210 & -0.273597 \\
\hline 10 & 7 & 0 & 0.334089 & 0.254146 & -0.976433 \\
\hline 11 & 1 & 0 & -0.435527 & -0.411298 & -1.078335 \\
\hline 12 & 7 & 0 & -2.323851 & -1.102598 & -0.186486 \\
\hline 13 & 1 & 0 & -3.259457 & -0.858904 & -0.507525 \\
\hline 14 & 1 & 0 & -2.106266 & -0.502630 & 0.609952 \\
\hline 15 & 1 & 0 & -2.369347 & -2.060376 & 0.157411 \\
\hline 16 & 9 & 0 & -0.501819 & 1.224930 & 1.055132 \\
\hline 17 & 1 & 0 & 2.327885 & 0.446135 & -0.455562 \\
\hline
\end{tabular}

Cis-2,3-Dichloroaziridine $+\mathrm{NH}_{3}$ Transition State (Gas Phase)

$\mathrm{E}=-1108.1976681$; Thermal Correction $=0.096893$. Unique imaginary frequency $=-673.9606$.

\begin{tabular}{|c|c|c|c|c|c|}
\hline \multirow{2}{*}{$\begin{array}{l}\text { Center } \\
\text { Number }\end{array}$} & \multirow{2}{*}{$\begin{array}{l}\text { Atomic } \\
\text { Number }\end{array}$} & \multirow{2}{*}{$\begin{array}{l}\text { Atomic } \\
\text { Type }\end{array}$} & \multicolumn{3}{|c|}{ Coordinates (Angstroms) } \\
\hline & & & $\mathrm{X}$ & $Y$ & Z \\
\hline 1 & 6 & 0 & -0.698130 & -0.126976 & 0.683849 \\
\hline 2 & 1 & 0 & -1.251105 & -0.161992 & 1.615720 \\
\hline 3 & 6 & 0 & 0.684466 & -0.583999 & 0.713756 \\
\hline 4 & 1 & 0 & -0.091961 & 2.297562 & 1.465924 \\
\hline 5 & 7 & 0 & 0.342730 & -1.882159 & 0.493599 \\
\hline 6 & 1 & 0 & 1.199505 & -0.288130 & 1.634921 \\
\hline 7 & 7 & 0 & -0.567333 & 1.892893 & 0.657462 \\
\hline 8 & 1 & 0 & 0.774632 & -2.485749 & 1.204292 \\
\hline 9 & 1 & 0 & -1.461301 & 2.365647 & 0.516524 \\
\hline 10 & 1 & 0 & 0.031363 & 2.024610 & -0.162179 \\
\hline 11 & 17 & 0 & -1.670774 & -0.316846 & -0.719168 \\
\hline 12 & 17 & 0 & 1.815072 & 0.342656 & -0.617200 \\
\hline
\end{tabular}

Cis-2,3-Dichloroaziridine $+\mathrm{NH}_{3}$ Ground State (Gas Phase)

$\mathrm{E}=-1108.2740541$; Thermal Correction $=0.099546$.

\begin{tabular}{|c|c|c|c|c|c|}
\hline \multirow{2}{*}{$\begin{array}{l}\text { Center } \\
\text { Number }\end{array}$} & \multirow{2}{*}{$\begin{array}{l}\text { Atomic } \\
\text { Number }\end{array}$} & \multirow{2}{*}{$\begin{array}{l}\text { Atomic } \\
\text { Type }\end{array}$} & \multicolumn{3}{|c|}{ Coordinates (Angstroms) } \\
\hline & & & $\mathrm{X}$ & $\mathrm{Y}$ & Z \\
\hline 1 & 7 & 0 & 3.772087 & -0.000153 & -0.071029 \\
\hline 2 & 1 & 0 & 4.083286 & -0.001093 & -1.041468 \\
\hline 3 & 1 & 0 & 4.185870 & -0.818250 & 0.373561 \\
\hline 4 & 1 & 0 & 4.185481 & 0.819069 & 0.371850 \\
\hline 5 & 6 & 0 & -0.017544 & -0.739691 & 0.473098 \\
\hline 6 & 7 & 0 & 0.751867 & -0.000124 & -0.497349 \\
\hline 7 & 6 & 0 & -0.017393 & 0.739597 & 0.473101 \\
\hline
\end{tabular}




\begin{tabular}{|c|c|c|c|c|c|}
\hline 8 & 1 & 0 & 0.488587 & -1.253016 & 1.287177 \\
\hline 9 & 1 & 0 & 1.766039 & -0.000225 & -0.315626 \\
\hline 10 & 1 & 0 & 0.488845 & 1.252813 & 1.287183 \\
\hline 11 & 17 & 0 & -1.372413 & -1.683462 & -0.107687 \\
\hline 12 & 17 & 0 & -1.372067 & 1.683650 & -0.107679 \\
\hline \multicolumn{6}{|c|}{$\begin{array}{l}\text { trans-2,3-Dichloroaziridine }+\mathrm{NH}_{3} \text { Transition State } \\
\mathrm{E}=-1108.2047756 ; \text { Thermal Correction }=0.097195 . \\
\text { frequency }=-664.8742 .\end{array}$} \\
\hline Center & Atomic & Atomic & \multicolumn{3}{|c|}{ Coordinates (Angstroms) } \\
\hline Number & Number & Type & $\mathrm{X}$ & $Y$ & $\mathrm{Z}$ \\
\hline 1 & 1 & 0 & 0.525777 & -0.033572 & 1.502248 \\
\hline 2 & 6 & 0 & 0.574738 & -0.053362 & 0.422644 \\
\hline 3 & 6 & 0 & -0.479798 & -0.761662 & -0.280073 \\
\hline 4 & 1 & 0 & 0.255918 & 2.166151 & -0.759048 \\
\hline 5 & 7 & 0 & -0.283173 & -1.844250 & 0.521346 \\
\hline 6 & 1 & 0 & -0.374101 & -0.823897 & -1.365606 \\
\hline 7 & 7 & 0 & 0.248942 & 1.909749 & 0.229263 \\
\hline 8 & 1 & 0 & -0.147478 & -2.693795 & -0.040936 \\
\hline 9 & 1 & 0 & 0.917362 & 2.498874 & 0.728641 \\
\hline 10 & 1 & 0 & -0.698513 & 2.053683 & 0.583828 \\
\hline 11 & 17 & 0 & 2.180817 & -0.110399 & -0.240997 \\
\hline 12 & 17 & 0 & -2.228405 & 0.184764 & -0.156581 \\
\hline \multicolumn{6}{|c|}{$\begin{array}{l}\text { trans-2,3-Dichloroaziridine }+\mathrm{NH}_{3} \text { Ground State (Gas Phase) } \\
\mathrm{E}=-1108.2774735 ; \text { Thermal Correction }=0.099426 .\end{array}$} \\
\hline Center & Atomic & Atomic & \multicolumn{3}{|c|}{ Coordinates (Angstroms) } \\
\hline Number & Number & Type & $\mathrm{X}$ & $Y$ & Z \\
\hline 1 & 6 & 0 & 0.069828 & -0.757024 & 0.534627 \\
\hline 2 & 6 & 0 & 0.817904 & 0.203254 & -0.291805 \\
\hline 3 & 1 & 0 & 0.561560 & -1.383788 & 1.270285 \\
\hline 4 & 7 & 0 & 0.012865 & 0.657590 & 0.819112 \\
\hline 5 & 1 & 0 & -0.854261 & 1.128240 & 0.509406 \\
\hline 6 & 17 & 0 & 2.561559 & 0.376868 & -0.162778 \\
\hline 7 & 1 & 0 & -2.699135 & 1.889453 & -1.155864 \\
\hline 8 & 7 & 0 & -2.574863 & 1.883677 & -0.144657 \\
\hline 9 & 1 & 0 & -3.287874 & 1.263327 & 0.236423 \\
\hline 10 & 1 & 0 & -2.783355 & 2.823795 & 0.188314 \\
\hline 11 & 17 & 0 & -1.314924 & -1.586851 & -0.185378 \\
\hline 12 & 1 & 0 & 0.477857 & 0.382424 & -1.308028 \\
\hline
\end{tabular}

$\underline{\mathbf{S}}_{\underline{N}} \underline{1}$ Reactions (See above for the ground state data in solution.) 
2-Fluoroaziridine Transition State (No Solvent) E= -232.5973645; Thermal Energy Correction $=0.063230$. Unique imaginary frequency $=-793.0818 \mathrm{~cm}^{-1}$.

\begin{tabular}{cccrrr} 
Center & $\begin{array}{c}\text { Atomic } \\
\text { Number }\end{array}$ & $\begin{array}{c}\text { Atomic } \\
\text { Number }\end{array}$ & \multicolumn{3}{c}{ Coordinates } \\
Type & X Angstroms) & \multicolumn{2}{c}{ Y } \\
-1 & 6 & 0 & -0.249021 & -0.058567 & 0.424296 \\
2 & 7 & 0 & -1.355813 & -0.345954 & -0.296541 \\
3 & 1 & 0 & -2.006875 & -0.909848 & 0.267187 \\
4 & 6 & 0 & 0.200350 & 1.174622 & -0.056073 \\
5 & 1 & 0 & 0.615272 & 1.955417 & 0.576908 \\
6 & 1 & 0 & 0.057632 & 1.357685 & -1.114480 \\
7 & 1 & 0 & -0.135010 & -0.330977 & 1.471710 \\
8 & 9 & 0 & 1.250188 & -0.705214 & -0.148320
\end{tabular}

2-Fluoroaziridine Transition State in THF $\quad \mathrm{E}=-232.6180910$; Thermal Energy Correction = 0.062123 . Unique imaginary frequency $=-615.2431 \mathrm{~cm}^{-1}$.

\begin{tabular}{|c|c|c|c|c|c|}
\hline \multirow{2}{*}{$\begin{array}{l}\text { Center } \\
\text { Number }\end{array}$} & \multirow{2}{*}{$\begin{array}{l}\text { Atomic } \\
\text { Number }\end{array}$} & \multirow{2}{*}{$\begin{array}{c}\text { Atomic } \\
\text { Type }\end{array}$} & \multicolumn{3}{|c|}{ Coordinates (Angstroms) } \\
\hline & & & $\mathrm{x}$ & $\mathrm{Y}$ & Z \\
\hline 1 & 6 & 0 & -0.283444 & -0.085107 & 0.432536 \\
\hline 2 & 7 & 0 & -1.294669 & -0.538700 & -0.313836 \\
\hline 3 & 1 & 0 & -1.894497 & -1.177991 & 0.247495 \\
\hline 4 & 9 & 0 & 1.455832 & -0.534195 & -0.132306 \\
\hline 5 & 6 & 0 & -0.050911 & 1.201987 & -0.063073 \\
\hline 6 & 1 & 0 & 0.131560 & 2.061459 & 0.582862 \\
\hline 7 & 1 & 0 & -0.152221 & 1.349174 & -1.134000 \\
\hline 8 & 1 & 0 & -0.118516 & -0.355269 & 1.474470 \\
\hline
\end{tabular}

2-Fluoroaziridine Transition State in AN; E = -232.6239979; Thermal Energy Correction = 0.061682 . Unique imaginary frequency $=-546.4893 \mathrm{~cm}^{-1}$.

\begin{tabular}{ccccrr}
$\begin{array}{c}\text { Center } \\
\text { Number }\end{array}$ & $\begin{array}{c}\text { Atomic } \\
\text { Number }\end{array}$ & $\begin{array}{c}\text { Atomic } \\
\text { Type }\end{array}$ & \multicolumn{2}{c}{ Coordinates (Angstroms) } \\
-1 & 6 & 0 & -0.305305 & -0.101446 & 0.440937 \\
1 & 7 & 0 & -1.270567 & -0.599830 & -0.324865 \\
2 & 1 & 0 & -1.864585 & -1.261680 & 0.225336 \\
3 & 6 & 0 & -0.152235 & 1.194202 & -0.064884 \\
4 & 1 & 0 & -0.079978 & 2.070600 & 0.582033 \\
5 & 1 & 0 & -0.218077 & 1.327299 & -1.140683 \\
6 & 1 & 0 & -0.120259 & -0.374490 & 1.479152 \\
7 & 9 & 0 & 1.546902 & -0.457717 & -0.125344 \\
8 & & 0 & & &
\end{tabular}

2-Fluoroaziridine Transition State in $\mathrm{H}_{2} \mathrm{O} ; \quad \mathrm{E}=-232.6472917$; Thermal Energy Correction = 0.061189 . Unique imaginary frequency $=-563.0312 \mathrm{~cm}^{-1}$.
1
6
0
$-0.754058$
0.681676
0.000744 


$\begin{array}{rllrrr}2 & 7 & 0 & -1.650182 & -0.208076 & -0.000757 \\ 3 & 1 & 0 & -2.697646 & -0.207060 & -0.002876 \\ 4 & 6 & 0 & 0.044281 & -0.532506 & 0.001031 \\ 5 & 1 & 0 & 0.295681 & -1.003821 & -0.937332 \\ 6 & 1 & 0 & 0.294082 & -1.004995 & 0.939235 \\ 7 & 9 & 0 & 2.067474 & 0.111668 & -0.000624 \\ 8 & 1 & 0 & -0.689447 & 1.772375 & 0.001239\end{array}$

2-Fluoroaziridine in $\mathrm{H}_{2} \mathrm{O}$; $\mathrm{E}=-232.7226811$; Thermal Energy Correction $=0.066251$.

\begin{tabular}{ccrrr}
$\begin{array}{c}\text { Center } \\
\text { Number }\end{array}$ & $\begin{array}{c}\text { Atomic } \\
\text { Number }\end{array}$ & $\begin{array}{c}\text { Atomic } \\
\text { Type }\end{array}$ & \multicolumn{2}{c}{ Coordinates } \\
X Angstroms)
\end{tabular}

2,2-Difluoroaziridine Transition State in $\mathrm{H}_{2} \mathrm{O} ; \quad \mathrm{E}=-331.7433025$; Thermal Energy Correction $=$ 0.054397 . Unique imaginary frequency $=-193.5657 \mathrm{~cm}^{-1}$.

\begin{tabular}{cccrrr}
$\begin{array}{c}\text { Center } \\
\text { Number }\end{array}$ & $\begin{array}{c}\text { Atomic } \\
\text { Number }\end{array}$ & $\begin{array}{c}\text { Atomic } \\
\text { Type }\end{array}$ & \multicolumn{2}{c}{ Coordinates (Angstroms) } \\
----1 & $\mathrm{X}$ & $\mathrm{Y}$ \\
1 & 6 & 0 & 0.676160 & 0.164966 & -0.000464 \\
2 & 7 & 0 & 1.417915 & -0.839311 & -0.000274 \\
3 & 1 & 0 & 2.401382 & -1.215096 & 0.000130 \\
4 & 6 & 0 & -0.248693 & -0.935398 & 0.000473 \\
5 & 1 & 0 & -0.627936 & -1.315093 & 0.939945 \\
6 & 1 & 0 & -0.628199 & -1.316317 & -0.938348 \\
7 & 9 & 0 & 0.787310 & 1.458493 & 0.000218 \\
8 & 9 & 0 & -2.302360 & 0.135315 & -0.000203
\end{tabular}

2-Chloroaziridine Transition State (No Solvent) $\quad \mathrm{E}=-592.6198448$; Thermal Energy Correction $=0.062502$. Unique imaginary frequency $=-824.1391 \mathrm{~cm}^{-1}$.

\begin{tabular}{|c|c|c|c|c|c|}
\hline \multirow{2}{*}{$\begin{array}{l}\text { Center } \\
\text { Number }\end{array}$} & \multirow{2}{*}{$\begin{array}{l}\text { Atomic } \\
\text { Number }\end{array}$} & \multirow{2}{*}{$\begin{array}{c}\text { Atomic } \\
\text { Type }\end{array}$} & \multicolumn{3}{|c|}{ Coordinates (Angstroms) } \\
\hline & & & $\mathrm{X}$ & $\mathrm{Y}$ & Z \\
\hline 1 & 6 & 0 & -0.676513 & -0.111147 & 0.441461 \\
\hline 2 & 7 & 0 & -1.569632 & -0.782020 & -0.333882 \\
\hline 3 & 1 & 0 & -2.102843 & -1.445455 & 0.247470 \\
\hline 4 & 17 & 0 & 1.428345 & -0.148967 & -0.057729 \\
\hline 5 & 6 & 0 & -0.798246 & 1.178033 & -0.079523 \\
\hline 6 & 1 & 0 & -0.909041 & 2.062709 & 0.545587 \\
\hline 7 & 1 & 0 & -0.872460 & 1.266776 & -1.155872 \\
\hline 8 & 1 & 0 & -0.561538 & -0.278774 & 1.509756 \\
\hline
\end{tabular}

2-Chloroaziridine Transition State in THF $E=-592.6624645$; Thermal Energy Correction $=$ 0.061226 . Unique imaginary frequency $=-549.4221 \mathrm{~cm}^{-1}$.

Center Atomic Atomic Coordinates (Angstroms) 


\begin{tabular}{|c|c|c|c|c|c|}
\hline Number & Number & Type & $\mathrm{X}$ & Y & Z \\
\hline 1 & 6 & 0 & 1.265325 & 0.662221 & 0.000231 \\
\hline 2 & 7 & 0 & 2.253896 & -0.122673 & -0.000344 \\
\hline 3 & 1 & 0 & 3.289348 & -0.001644 & 0.000068 \\
\hline 4 & 17 & 0 & -1.878144 & 0.062816 & -0.000063 \\
\hline 5 & 6 & 0 & 0.575787 & -0.615943 & 0.000229 \\
\hline 6 & 1 & 0 & 0.369897 & -1.111329 & 0.938653 \\
\hline 7 & 1 & 0 & 0.369645 & -1.111113 & -0.938220 \\
\hline 8 & 1 & 0 & 1.075620 & 1.737259 & 0.000222 \\
\hline
\end{tabular}

2-Chloroaziridine Transition State in AN $E=-592.6702751$; Thermal Energy Correction $=$ 0.060799 . Unique imaginary frequency $=-601.1990 \mathrm{~cm}^{-1}$.

\begin{tabular}{|c|c|c|c|c|c|}
\hline \multirow{2}{*}{$\begin{array}{l}\text { Center } \\
\text { Number }\end{array}$} & \multirow{2}{*}{$\begin{array}{l}\text { Atomic } \\
\text { Number }\end{array}$} & \multirow{2}{*}{$\begin{array}{c}\text { Atomic } \\
\text { Type }\end{array}$} & \multicolumn{3}{|c|}{ Coordinates (Angstroms) } \\
\hline & & & $\mathrm{X}$ & $\mathrm{Y}$ & Z \\
\hline 1 & 6 & 0 & -1.271519 & 0.652028 & 0.000308 \\
\hline 2 & 7 & 0 & -2.261078 & -0.130986 & -0.000267 \\
\hline 3 & 1 & 0 & -3.297251 & 0.023653 & -0.001119 \\
\hline 4 & 17 & 0 & 1.871625 & 0.065773 & -0.000099 \\
\hline 5 & 6 & 0 & -0.544110 & -0.607562 & 0.000313 \\
\hline 6 & 1 & 0 & -0.354320 & -1.110532 & -0.937997 \\
\hline 7 & 1 & 0 & -0.355065 & -1.111079 & 0.938455 \\
\hline 8 & 1 & 0 & -1.089667 & 1.729914 & 0.000491 \\
\hline
\end{tabular}

2-Chloroaziridine Transition State in $\mathrm{H}_{2} \mathrm{O} \quad \mathrm{E}=-592.6714692$; Thermal Energy Correction = 0.060726 . Unique imaginary frequency $=-608.5946 \mathrm{~cm}^{-1}$.

\begin{tabular}{|c|c|c|c|c|c|}
\hline \multirow{2}{*}{$\begin{array}{l}\text { Center } \\
\text { Number }\end{array}$} & Atomic & \multirow{2}{*}{$\begin{array}{c}\text { Atomic } \\
\text { Type }\end{array}$} & \multicolumn{3}{|c|}{ Coordinates (Angstroms) } \\
\hline & Number & & $\mathrm{X}$ & $\mathrm{Y}$ & Z \\
\hline 1 & 6 & 0 & -0.091199 & 0.685473 & 0.342728 \\
\hline 2 & 6 & 0 & -1.571695 & -0.755065 & 0.145660 \\
\hline 3 & 1 & 0 & 0.156037 & 1.599100 & 0.885260 \\
\hline 4 & 1 & 0 & -1.710788 & -1.592610 & -0.536583 \\
\hline 5 & 1 & 0 & -1.722050 & -0.872151 & 1.211582 \\
\hline 6 & 7 & 0 & -1.237198 & 0.509138 & -0.319730 \\
\hline 7 & 1 & 0 & -1.751213 & 1.157044 & -0.941086 \\
\hline 8 & 17 & 0 & 1.392104 & -0.202223 & -0.077141 \\
\hline
\end{tabular}

2,2-Dichloroaziridine Transition State (No Solvent) $\quad E=-592.6198448$; Thermal Energy Correction $=0.062502$. Unique imaginary frequency $=-824.1391 \mathrm{~cm}^{-1}$.

\begin{tabular}{|c|c|c|c|}
\hline Center & Atomic & Atomic & Coordinates \\
\hline Number & Number & Type & $X \quad Y$ \\
\hline
\end{tabular}




$\begin{array}{rrrrrr}1 & 6 & 0 & -0.245010 & 0.454509 & -0.066161 \\ 2 & 7 & 0 & -0.426077 & 1.498815 & -0.894404 \\ 3 & 1 & 0 & -1.308699 & 1.411466 & -1.416678 \\ 4 & 17 & 0 & 1.649695 & -0.571678 & -0.162836 \\ 5 & 17 & 0 & -1.417715 & -0.840220 & 0.069831 \\ 6 & 6 & 0 & 0.190177 & 1.098352 & 1.100293 \\ 7 & 1 & 0 & -0.263985 & 0.914794 & 2.072916 \\ 8 & 1 & 0 & 0.940565 & 1.867133 & 0.980874\end{array}$

2,2-Dichloroaziridine Transition State in THF $E=-1051.7701514$; Thermal Energy Correction $=0.053261$. Unique imaginary frequency $=-463.6362 \mathrm{~cm}^{-1}$.

\begin{tabular}{cccrrr} 
Center & Atomic & Atomic & \multicolumn{3}{c}{ Coordinates (Angstroms) } \\
Number & Number & Type & \multicolumn{2}{c}{ X } & \multicolumn{1}{c}{ Y } \\
-1 & 6 & 0 & -0.938354 & 0.361298 & -0.001028 \\
2 & 7 & 0 & -1.537978 & 1.468880 & -0.001226 \\
3 & 1 & 0 & -2.511687 & 1.846550 & -0.001019 \\
4 & 17 & 0 & 2.278401 & -0.224633 & -0.000455 \\
5 & 17 & 0 & -1.299181 & -1.261997 & 0.000328 \\
6 & 6 & 0 & 0.184365 & 1.282160 & 0.001925 \\
7 & 1 & 0 & 0.576030 & 1.639892 & 0.944208 \\
8 & 1 & 0 & 0.578689 & 1.643360 & -0.937832
\end{tabular}

2,2-Dichloroaziridine Transition State in AN E = -1051.7783733; Thermal Energy Correction = 0.052817 . Unique imaginary frequency $=-527.7091 \mathrm{~cm}^{-1}$.

\begin{tabular}{cccrrr}
$\begin{array}{c}\text { Center } \\
\text { Number }\end{array}$ & $\begin{array}{c}\text { Atomic } \\
\text { Number }\end{array}$ & $\begin{array}{c}\text { Atomic } \\
\text { Type }\end{array}$ & \multicolumn{2}{c}{ Coordinates (Angstroms) } \\
-1 & $\mathbf{X}$ & \multicolumn{1}{c}{ Y } & $\mathrm{Z}$ \\
\hline 1 & 6 & 0 & -0.934458 & 0.371522 & -0.000457 \\
2 & 7 & 0 & -1.546712 & 1.469615 & -0.000406 \\
3 & 1 & 0 & -2.539916 & 1.811795 & 0.001776 \\
4 & 17 & 0 & 2.263169 & -0.223004 & -0.000048 \\
5 & 17 & 0 & -1.291015 & -1.259311 & 0.000073 \\
6 & 6 & 0 & 0.212113 & 1.264461 & 0.000331 \\
7 & 1 & 0 & 0.587056 & 1.641410 & 0.942320 \\
8 & 1 & 0 & 0.587303 & 1.642954 & -0.940916
\end{tabular}

2,2-Dichloroaziridine Transition State in $\mathrm{H}_{2} \mathrm{O} \quad \mathrm{E}=-1051.7797666$; Thermal Energy Correction = 0.052746 . Unique imaginary frequency $=-537.0083 \mathrm{~cm}^{-1}$.

\begin{tabular}{llcl} 
Center & Atomic & Atomic & \multicolumn{2}{c}{ Coordinates (Angstroms) } \\
Number & Number & Type & $\mathrm{X}$
\end{tabular}




$\begin{array}{rrrrrr}1 & 6 & 0 & -0.934162 & 0.373054 & -0.000516 \\ 2 & 7 & 0 & -1.548747 & 1.469199 & -0.000455 \\ 3 & 1 & 0 & -2.545030 & 1.805418 & 0.001296 \\ 4 & 17 & 0 & 2.260866 & -0.222365 & -0.000097 \\ 5 & 17 & 0 & -1.289069 & -1.259202 & 0.000106 \\ 6 & 6 & 0 & 0.215923 & 1.262051 & 0.000517 \\ 7 & 1 & 0 & 0.587494 & 1.642373 & 0.942586 \\ 8 & 1 & 0 & 0.587644 & 1.643822 & -0.940855\end{array}$

2-Bromoaziridine Transition State (No Solvent) $E=-2704.8335661$; Thermal Energy Correction $=0.062195$. Unique imaginary frequency $=-352.7944 \mathrm{~cm}^{-1}$.

\begin{tabular}{|c|c|c|c|c|c|}
\hline \multirow{2}{*}{$\begin{array}{l}\text { Center } \\
\text { Number }\end{array}$} & \multirow{2}{*}{$\begin{array}{l}\text { Atomic } \\
\text { Number }\end{array}$} & \multirow{2}{*}{$\begin{array}{l}\text { Atomic } \\
\text { Type }\end{array}$} & \multicolumn{3}{|c|}{ Coordinates (Angstroms) } \\
\hline & & & $\mathrm{X}$ & $\mathrm{Y}$ & Z \\
\hline 1 & 6 & 0 & -2.155246 & 0.714517 & -0.119203 \\
\hline 2 & 6 & 0 & -1.662414 & -0.584348 & -0.568113 \\
\hline 3 & 1 & 0 & -1.487715 & 1.558830 & -0.278849 \\
\hline 4 & 1 & 0 & -3.210338 & 0.896428 & 0.046581 \\
\hline 5 & 7 & 0 & -1.602435 & -0.428870 & 0.696001 \\
\hline 6 & 1 & 0 & -0.700970 & -0.523811 & 1.234434 \\
\hline 7 & 35 & 0 & 1.165930 & 0.044319 & -0.012250 \\
\hline 8 & 1 & 0 & -1.285519 & -1.261541 & -1.321537 \\
\hline
\end{tabular}

2-Bromoaziridine Transition State in THF $E=-2704.8640811$; Thermal Energy Correction = 0.061048 . Unique imaginary frequency $=-543.9095 \mathrm{~cm}^{-1}$.

\begin{tabular}{cccrrr}
$\begin{array}{c}\text { Center } \\
\text { Number }\end{array}$ & $\begin{array}{c}\text { Atomic } \\
\text { Number }\end{array}$ & $\begin{array}{c}\text { Atomic } \\
\text { Type }\end{array}$ & \multicolumn{2}{c}{$\begin{array}{c}\text { Coordinates } \\
\text { (Angstroms) }\end{array}$} \\
-1 & 6 & 0 & -1.916153 & 0.659523 & -0.000013 \\
1 & 7 & 0 & -2.921478 & -0.103103 & -0.000888 \\
3 & 1 & 0 & -3.955115 & 0.037813 & 0.001517 \\
4 & 6 & 0 & -1.250919 & -0.632711 & 0.000616 \\
5 & 1 & 0 & -1.055947 & -1.133305 & -0.938024 \\
6 & 1 & 0 & -1.055756 & -1.132038 & 0.939910 \\
7 & 1 & 0 & -1.701889 & 1.730268 & 0.001568 \\
8 & 35 & 0 & 1.349185 & 0.030232 & -0.000068
\end{tabular}

2-Bromoaziridine Transition State in AN E = -2705.8715026; Thermal Energy Correction = 0.060606 . Unique imaginary frequency $=-594.2784 \mathrm{~cm}^{-1}$.

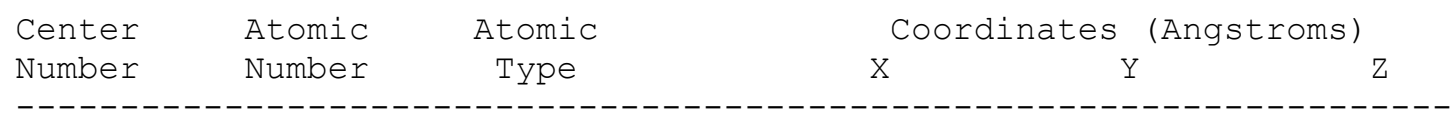




$\begin{array}{rrrrrr}1 & 6 & 0 & -1.009044 & 0.911015 & -0.000153 \\ 2 & 7 & 0 & -1.454409 & 2.087417 & -0.000636 \\ 3 & 1 & 0 & -2.386235 & 2.575619 & 0.000024 \\ 4 & 6 & 0 & 0.256239 & 1.631787 & 0.000727 \\ 5 & 1 & 0 & 0.680643 & 1.952947 & 0.943270 \\ 6 & 1 & 0 & 0.681565 & 1.953643 & -0.941100 \\ 7 & 35 & 0 & -1.684922 & -0.787938 & 0.000049 \\ 8 & 35 & 0 & 2.134114 & -0.250660 & -0.000083\end{array}$

2-Bromoaziridine Transition State in $\mathrm{H}_{2} \mathrm{O} \quad \mathrm{E}=-2705.8726250$; Thermal Energy Correction = 0.060532 . Unique imaginary frequency $=-602.0167 \mathrm{~cm}^{-1}$.

\begin{tabular}{|c|c|c|c|c|c|}
\hline \multirow{2}{*}{$\begin{array}{l}\text { Center } \\
\text { Number }\end{array}$} & \multirow{2}{*}{$\begin{array}{l}\text { Atomic } \\
\text { Number }\end{array}$} & \multirow{2}{*}{$\begin{array}{c}\text { Atomic } \\
\text { Type }\end{array}$} & \multicolumn{3}{|c|}{ Coordinates (Angstroms) } \\
\hline & & & $\mathrm{X}$ & $\mathrm{Y}$ & Z \\
\hline 1 & 6 & 0 & 1.919724 & 0.648474 & -0.000054 \\
\hline 2 & 7 & 0 & 2.926165 & -0.111855 & -0.000186 \\
\hline 3 & 1 & 0 & 3.960289 & 0.065701 & 0.000589 \\
\hline 4 & 6 & 0 & 1.212604 & -0.624353 & 0.000110 \\
\hline 5 & 1 & 0 & 1.036299 & -1.132768 & 0.938926 \\
\hline 6 & 1 & 0 & 1.036474 & -1.133107 & -0.938531 \\
\hline 7 & 1 & 0 & 1.713624 & 1.722517 & 0.000334 \\
\hline 8 & 35 & 0 & -1.343537 & 0.031883 & -0.000010 \\
\hline
\end{tabular}

2,2-Dibromoaziridine Transition State (No Solvent) $\quad E=-5278.1314775$; Thermal Energy Correction $=0.054049$. Unique imaginary frequency $=-405.1810 \mathrm{~cm}^{-1}$.

\begin{tabular}{|c|c|c|c|c|c|}
\hline \multirow{2}{*}{$\begin{array}{l}\text { Center } \\
\text { Number }\end{array}$} & \multirow{2}{*}{$\begin{array}{l}\text { Atomic } \\
\text { Number }\end{array}$} & \multirow{2}{*}{$\begin{array}{c}\text { Atomic } \\
\text { Type }\end{array}$} & \multicolumn{3}{|c|}{ Coordinates (Angstroms) } \\
\hline & & & X & $\mathrm{Y}$ & Z \\
\hline 1 & 6 & 0 & -0.069325 & 2.041994 & -0.597682 \\
\hline 2 & 6 & 0 & -0.693536 & 0.868996 & -0.001191 \\
\hline 3 & 1 & 0 & 0.816651 & 1.851249 & -1.199065 \\
\hline 4 & 1 & 0 & -0.617531 & 2.970597 & -0.716747 \\
\hline 5 & 7 & 0 & -0.014172 & 1.557456 & 0.846257 \\
\hline 6 & 1 & 0 & 0.824795 & 1.186914 & 1.349016 \\
\hline 7 & 35 & 0 & -1.811065 & -0.535240 & -0.020185 \\
\hline 8 & 35 & 0 & 1.915421 & -0.446956 & -0.030208 \\
\hline
\end{tabular}

2,2-Dibromoaziridine Transition State in THF $E=-5278.1314775$; Thermal Energy Correction $=0.054049$. Unique imaginary frequency $=-405.1810 \mathrm{~cm}^{-1}$.

\begin{tabular}{|c|c|c|c|c|c|}
\hline \multirow{2}{*}{$\begin{array}{l}\text { Center } \\
\text { Number }\end{array}$} & \multirow{2}{*}{$\begin{array}{l}\text { Atomic } \\
\text { Number }\end{array}$} & \multirow{2}{*}{$\begin{array}{c}\text { Atomic } \\
\text { Type }\end{array}$} & \multicolumn{3}{|c|}{ Coordinates (Angstroms) } \\
\hline & & & $\mathrm{x}$ & $Y$ & Z \\
\hline $\begin{array}{l}1 \\
2\end{array}$ & $\begin{array}{l}6 \\
7\end{array}$ & $\begin{array}{l}0 \\
0\end{array}$ & $\begin{array}{l}1.015096 \\
1.451574\end{array}$ & $\begin{array}{l}0.901482 \\
2.083724\end{array}$ & $\begin{array}{l}-0.000119 \\
-0.000048\end{array}$ \\
\hline
\end{tabular}




$\begin{array}{rrrrrr}3 & 1 & 0 & 2.364191 & 2.594083 & -0.000110 \\ 4 & 6 & 0 & -0.229783 & 1.655380 & 0.000126 \\ 5 & 1 & 0 & -0.667669 & 1.959244 & -0.941427 \\ 6 & 1 & 0 & -0.667448 & 1.958996 & 0.941856 \\ 7 & 35 & 0 & 1.689073 & -0.790262 & 0.00012 \\ 8 & 35 & 0 & -2.143415 & -0.250869 & -0.000013\end{array}$

2,2-Dibromoaziridine Transition State in AN E = -5278.1709618; Thermal Energy Correction= 0.052187 . Unique imaginary frequency $=-540.4936 \mathrm{~cm}^{-1}$.

\begin{tabular}{cccrrr}
$\begin{array}{c}\text { Center } \\
\text { Number }\end{array}$ & $\begin{array}{c}\text { Atomic } \\
\text { Number }\end{array}$ & $\begin{array}{c}\text { Atomic } \\
\text { Type }\end{array}$ & \multicolumn{2}{c}{ Coordinates (Angstroms) } \\
-1 & 6 & 0 & -1.008919 & 0.910702 & 0.000023 \\
1 & 7 & 0 & -1.453608 & 2.087436 & -0.000296 \\
2 & 1 & 0 & -2.385033 & 2.576147 & -0.001191 \\
3 & 6 & 0 & 0.256274 & 1.631545 & 0.000518 \\
4 & 1 & 0 & 0.681548 & 1.952175 & 0.942838 \\
5 & 1 & 0 & 0.682034 & 1.952214 & -0.941558 \\
6 & 35 & 0 & 2.134281 & -0.250804 & -0.000068 \\
7 & 35 & 0 & -1.685350 & -0.787656 & 0.000031 \\
8 & & & & &
\end{tabular}

2,2-Dibromoaziridine Transition State in $\mathrm{H}_{2} \mathrm{O} \quad \mathrm{E}=-5278.1724071$; Thermal Energy Correction = 0.052110 . Unique imaginary frequency $=-552.4614 \mathrm{~cm}^{-1}$.

\section{$S_{\mathrm{N}} 1$ Ground State Energies in Cyclohexane:}

2,2-Difluoroaziridine $\mathrm{E}=-331.8206190$; Thermal Energy Correction $=0.05247$.

\begin{tabular}{cccrrr}
$\begin{array}{c}\text { Center } \\
\text { Number }\end{array}$ & $\begin{array}{c}\text { Atomic } \\
\text { Number }\end{array}$ & $\begin{array}{c}\text { Atomic } \\
\text { Type }\end{array}$ & \multicolumn{2}{c}{ Coordinates (Angstroms) } \\
-1 & 6 & 0 & X & $Z$ \\
1 & 9 & 0 & 0.144404 & -0.018393 & -0.022242 \\
2 & 6 & 0 & -1.156521 & -0.123345 & -0.662223 \\
3 & 7 & 0 & -0.951382 & -0.097761 & 0.848072 \\
4 & 1 & 0 & -1.550830 & 0.769901 & -1.138409 \\
5 & 1 & 0 & -1.184692 & 0.812157 & 1.259925 \\
6 & 1 & 0 & -1.508122 & -1.078367 & -1.035738 \\
7 & 9 & 0 & 1.041115 & -1.038830 & 0.006080 \\
8 & & 0 & & &
\end{tabular}

2-Chloroaziridine $\mathrm{E}=-592.7254039$; Thermal Energy Correction $=0.066194$.

\begin{tabular}{|c|c|c|c|c|c|}
\hline \multirow{2}{*}{$\begin{array}{l}\text { Center } \\
\text { Number }\end{array}$} & \multirow{2}{*}{$\begin{array}{l}\text { Atomic } \\
\text { Number }\end{array}$} & \multirow{2}{*}{$\begin{array}{l}\text { Atomic } \\
\text { Type }\end{array}$} & \multicolumn{3}{|c|}{ Coordinates (Angstroms) } \\
\hline & & & $\mathrm{X}$ & Y & Z \\
\hline 1 & 6 & 0 & 0.262783 & -0.106343 & 0.507872 \\
\hline 2 & 6 & 0 & 1.342383 & 0.722572 & -0.056585 \\
\hline 3 & 1 & 0 & 1.120416 & 1.383329 & -0.887733 \\
\hline
\end{tabular}




$\begin{array}{rrrrrr}4 & 1 & 0 & 2.136042 & 1.047819 & 0.611776 \\ 5 & 7 & 0 & 1.219881 & -0.717189 & -0.386677 \\ 6 & 1 & 0 & 1.887947 & -1.296956 & 0.130636 \\ 7 & 17 & 0 & -1.387613 & 0.032529 & -0.083449 \\ 8 & 1 & 0 & 0.274857 & -0.364239 & 1.562972\end{array}$

2-Bromoaziridine $\mathrm{E}=-2705.917000$; Thermal Energy Correction $=0.065688$.

\begin{tabular}{cccrrr}
$\begin{array}{c}\text { Center } \\
\text { Number }\end{array}$ & $\begin{array}{c}\text { Atomic } \\
\text { Number }\end{array}$ & $\begin{array}{c}\text { Atomic } \\
\text { Type }\end{array}$ & \multicolumn{2}{c}{ Coordinates (Angstroms) } \\
---1 & 0 & -0.817290 & -0.042404 & 0.592918 \\
1 & 6 & 0 & -1.841419 & 0.696899 & -0.166484 \\
2 & 6 & 0 & -0.835763 & -0.134983 & 1.673694 \\
3 & 1 & 0 & -1.518483 & 1.202921 & -1.071998 \\
4 & 1 & 0 & -2.685695 & 1.141631 & 0.350914 \\
5 & 1 & 0 & -1.807854 & -0.791534 & -0.143194 \\
6 & 7 & 0 & -1.434731 & -1.170256 & -1.019325 \\
7 & 1 & 0 & 1.002340 & 0.016413 & -0.042558 \\
8 & 35 & & & &
\end{tabular}

2,2-Dibromoaziridine $\mathrm{E}=-5278.2126788$; Thermal Energy Correction $=0.056258$.

\begin{tabular}{cccrrr}
$\begin{array}{c}\text { Center } \\
\text { Number }\end{array}$ & $\begin{array}{c}\text { Atomic } \\
\text { Number }\end{array}$ & $\begin{array}{c}\text { Atomic } \\
\text { Type }\end{array}$ & \multicolumn{2}{c}{ Coordinates (Angstroms) } \\
-1 & $\mathbf{X}$ & \multicolumn{1}{c}{ Y } & Z \\
1 & 6 & 0 & 0.002856 & 0.580354 & 0.007525 \\
2 & 6 & 0 & -0.045349 & 1.879720 & -0.682061 \\
3 & 1 & 0 & 0.857366 & 2.272109 & -1.139707 \\
4 & 1 & 0 & -0.989671 & 2.166275 & -1.138227 \\
5 & 7 & 0 & 0.026714 & 1.759576 & 0.807916 \\
6 & 1 & 0 & -0.869761 & 1.942864 & 1.272902 \\
7 & 35 & 0 & 1.615267 & -0.455038 & -0.002990 \\
8 & 35 & 0 & -1.584695 & -0.500925 & -0.014244
\end{tabular}

Ammonia $\mathrm{E}=-56.4375012 ;$ Thermal Correction $=0.037751$.

\begin{tabular}{|c|c|c|c|c|c|}
\hline \multirow{2}{*}{$\begin{array}{l}\text { Center } \\
\text { Number }\end{array}$} & \multirow{2}{*}{$\begin{array}{l}\text { Atomic } \\
\text { Number }\end{array}$} & \multirow{2}{*}{$\begin{array}{l}\text { Atomic } \\
\text { Type }\end{array}$} & \multicolumn{3}{|c|}{ Coordinates (Angstroms) } \\
\hline & & & $\mathrm{x}$ & $Y$ & Z \\
\hline 1 & 7 & 0 & 0.000000 & 0.000000 & 0.111247 \\
\hline 2 & 1 & 0 & 0.000000 & 0.948438 & -0.259577 \\
\hline 3 & 1 & 0 & -0.821371 & -0.474219 & -0.259577 \\
\hline 4 & 1 & 0 & 0.821371 & -0.474219 & -0.259577 \\
\hline
\end{tabular}

\section{$S_{N} 2$ Ground State Data in Cyclohexane:}

2-Chloroaziridine E= -592.7254039; Thermal Energy Correction $=0.066194$.

\begin{tabular}{|c|c|c|c|c|c|}
\hline Center & Atomic & Atomic & \multicolumn{3}{|c|}{ Coordinates (Angstroms) } \\
\hline Number & Number & Type & $\mathrm{x}$ & $Y$ & Z \\
\hline 1 & 6 & 0 & 0.262783 & -0.106343 & 0.507872 \\
\hline
\end{tabular}




$\begin{array}{rrrrrr}2 & 6 & 0 & 1.342383 & 0.722572 & -0.056585 \\ 3 & 1 & 0 & 1.120416 & 1.383329 & -0.887733 \\ 4 & 1 & 0 & 2.136042 & 1.047819 & 0.611776 \\ 5 & 7 & 0 & 1.219881 & -0.717189 & -0.386677 \\ 6 & 1 & 0 & 1.887947 & -1.296956 & 0.130636 \\ 7 & 17 & 0 & -1.387613 & 0.032529 & -0.083449 \\ 8 & 1 & 0 & 0.274857 & -0.364239 & 1.562972\end{array}$

2-Bromoaziridine $E=-2762.3634841$; Thermal Energy Correction $=0.107050$.

\begin{tabular}{cccrrr}
$\begin{array}{c}\text { Center } \\
\text { Number }\end{array}$ & $\begin{array}{c}\text { Atomic } \\
\text { Number }\end{array}$ & $\begin{array}{c}\text { Atomic } \\
\text { Type }\end{array}$ & \multicolumn{2}{c}{ Coordinates (Angstroms) } \\
-0 & 6 & 0 & -0.494352 & 1.872970 & 0.644889 \\
1 & 6 & 0 & 0.264965 & 1.174208 & -0.406809 \\
2 & 1 & 0 & -0.633540 & 1.347189 & 1.586426 \\
3 & 1 & 0 & -0.470688 & 2.957065 & 0.707219 \\
4 & 7 & 0 & -1.151708 & 1.316081 & -0.567102 \\
5 & 1 & 0 & -1.668110 & 0.447825 & -0.349246 \\
6 & 1 & 0 & -2.030930 & -1.946795 & -0.648050 \\
7 & 7 & 0 & -2.468801 & -1.338773 & 0.044433 \\
8 & 1 & 0 & -2.202881 & -1.700444 & 0.960526 \\
9 & 1 & 0 & -3.477866 & -1.463116 & -0.043933 \\
10 & 35 & 0 & 1.036928 & -0.555659 & 0.032352 \\
11 & 1 & 0 & 0.911430 & 1.682135 & -1.115075
\end{tabular}

2,2-Dibromoaziridine E= -5334.6612220; Thermal Energy Correction $=0.097600$.

\begin{tabular}{ccccrr}
$\begin{array}{c}\text { Center } \\
\text { Number }\end{array}$ & $\begin{array}{c}\text { Atomic } \\
\text { Number }\end{array}$ & $\begin{array}{c}\text { Atomic } \\
\text { Type }\end{array}$ & \multicolumn{3}{c}{ Coordinates (Angstroms) } \\
-1 & 6 & 0 & -0.149240 & 0.386961 & 0.278460 \\
1 & 6 & 0 & 0.197368 & 1.260516 & 1.409483 \\
3 & 1 & 0 & -0.576642 & 1.859914 & 1.879899 \\
4 & 1 & 0 & 1.023851 & 0.945505 & 2.043308 \\
5 & 7 & 0 & 0.486844 & 1.611836 & -0.013256 \\
6 & 1 & 0 & 1.489900 & 1.513802 & -0.263919 \\
7 & 35 & 0 & -1.976961 & 0.199959 & -0.291833 \\
8 & 35 & 0 & 0.828606 & -1.273456 & 0.113602 \\
9 & 1 & 0 & 3.349740 & 0.633070 & -1.535925 \\
10 & 7 & 0 & 3.349687 & 1.234001 & -0.711016 \\
11 & 1 & 0 & 3.881315 & 2.071224 & -0.952669 \\
12 & 1 & 0 & 3.879778 & 0.743135 & 0.009633
\end{tabular}

\section{$S_{N} 1$ Transition State Data in Cyclohexane:}

2,2-Difluoroaziridine $\mathrm{E}=-331.7098163$; Thermal Energy Correction $=0.054866$. Unique imaginary frequency $=-791.2998 \mathrm{~cm}^{-1}$.

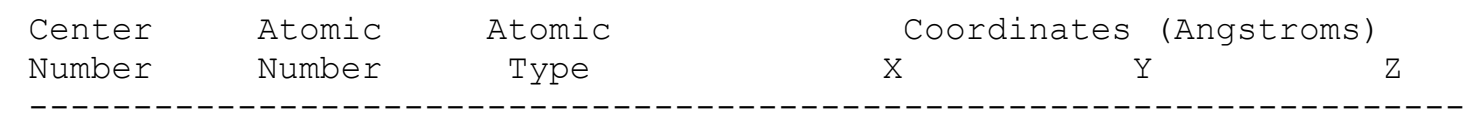




$\begin{array}{rrrrrr}1 & 6 & 0 & 0.222509 & -0.061646 & 0.025687 \\ 2 & 7 & 0 & 1.068693 & -0.834161 & -0.630315 \\ 3 & 1 & 0 & 1.951460 & -0.331975 & -0.819613 \\ 4 & 9 & 0 & -1.233763 & 0.386321 & -0.773197 \\ 5 & 6 & 0 & -0.567747 & -0.847144 & 0.875407 \\ 6 & 1 & 0 & -0.812799 & -0.543200 & 1.891311 \\ 7 & 1 & 0 & -0.877211 & -1.814310 & 0.499225 \\ 8 & 9 & 0 & 0.603667 & 1.167162 & 0.488166\end{array}$

2-Chloroaziridine $E=-592.6372781$; Thermal Energy Correction $=0.062380$. Unique imaginary frequency $=-112.5046 \mathrm{~cm}^{-1}$.

\begin{tabular}{cccrrr}
$\begin{array}{c}\text { Center } \\
\text { Number }\end{array}$ & $\begin{array}{c}\text { Atomic } \\
\text { Number }\end{array}$ & $\begin{array}{c}\text { Atomic } \\
\text { Type }\end{array}$ & \multicolumn{2}{c}{ Coordinates (Angstroms) } \\
-1 & \multicolumn{1}{c}{ X } & \multicolumn{1}{c}{$\mathrm{Y}$} \\
\hline 1 & 6 & 0 & 1.166904 & 0.685066 & 0.000562 \\
2 & 7 & 0 & 2.218606 & -0.015802 & -0.000560 \\
3 & 1 & 0 & 3.246540 & 0.061354 & -0.001819 \\
4 & 17 & 0 & -1.902609 & 0.050591 & -0.000175 \\
5 & 6 & 0 & 0.776917 & -0.709763 & 0.000514 \\
6 & 1 & 0 & 0.529942 & -1.193014 & 0.936150 \\
7 & 1 & 0 & 0.528782 & -1.192631 & -0.934968 \\
8 & 1 & 0 & 0.845919 & 1.723042 & 0.001071
\end{tabular}

2-Bromoaziridine $E=-2705.8403953$; Thermal Energy Correction $=0.062183$. Unique imaginary frequency $=-182.9500 \mathrm{~cm}^{-1}$.

\begin{tabular}{cccrrr} 
Center & $\begin{array}{c}\text { Atomic } \\
\text { Number }\end{array}$ & $\begin{array}{c}\text { Atomic } \\
\text { Number }\end{array}$ & \multicolumn{3}{c}{ Coordinates } \\
Type & X Angstroms) & \multicolumn{1}{c}{ Y } \\
-1 & 6 & 0 & -1.836669 & 0.680462 & 0.000349 \\
2 & 7 & 0 & -2.891877 & -0.014591 & -0.000426 \\
3 & 1 & 0 & -3.918778 & 0.082245 & -0.001040 \\
4 & 6 & 0 & -1.428779 & -0.709816 & 0.000348 \\
5 & 1 & 0 & -1.192980 & -1.196944 & -0.936294 \\
6 & 1 & 0 & -1.194207 & -1.197344 & 0.937091 \\
7 & 1 & 0 & -1.514923 & 1.718799 & 0.000855 \\
8 & 35 & 0 & 1.361620 & 0.024900 & -0.000052
\end{tabular}

2,2-Dibromoaziridine $E=-5278.1365236$; Thermal Energy Correction $=0.054384$. Unique imaginary frequency $=-116.6583 \mathrm{~cm}^{-1}$.

\begin{tabular}{|c|c|c|c|c|c|}
\hline Center & Atomic & Atomic & \multicolumn{3}{|c|}{ Coordinates (Angstroms) } \\
\hline Number & Number & Type & $\mathrm{x}$ & $\mathrm{Y}$ & Z \\
\hline 1 & 6 & 0 & -0.149240 & 0.386961 & 0.278460 \\
\hline 2 & 6 & 0 & 0.197368 & 1.260516 & 1.409483 \\
\hline 3 & 1 & 0 & -0.576642 & 1.859914 & 1.879899 \\
\hline 4 & 1 & 0 & 1.023851 & 0.945505 & 2.043308 \\
\hline
\end{tabular}




$\begin{array}{rrrrrr}5 & 7 & 0 & 0.486844 & 1.611836 & -0.013256 \\ 6 & 1 & 0 & 1.489900 & 1.513802 & -0.263919 \\ 7 & 35 & 0 & -1.976961 & 0.199959 & -0.291833 \\ 8 & 35 & 0 & 0.828606 & -1.273456 & 0.113602 \\ 9 & 1 & 0 & 3.349740 & 0.633070 & -1.535925 \\ 10 & 7 & 0 & 3.349687 & 1.234001 & -0.711016 \\ 11 & 1 & 0 & 3.881315 & 2.071224 & -0.952669 \\ 12 & 1 & 0 & 3.879778 & 0.743135 & 0.009633\end{array}$

\section{$\mathrm{S}_{\mathrm{N}} 2$ Transition State Data in Cyclohexane:}

2-Chloroaziridine $\mathrm{E}=-649.1091003$; Thermal Energy Correction $=0.105213$. Unique imaginary frequency $=-635.9719 \mathrm{~cm}^{-1}$.

\begin{tabular}{cccrrr}
$\begin{array}{c}\text { Center } \\
\text { Number }\end{array}$ & $\begin{array}{c}\text { Atomic } \\
\text { Number }\end{array}$ & $\begin{array}{c}\text { Atomic } \\
\text { Type }\end{array}$ & \multicolumn{3}{c}{ Coordinates (Angstroms) } \\
-1 & 6 & 0 & -0.714193 & 1.044163 & -0.156944 \\
-1 & 6 & 0 & 0.509786 & 0.563953 & 0.452179 \\
2 & 7 & 0 & -2.183061 & -0.319353 & 0.009816 \\
3 & 7 & 0 & 1.279146 & 1.582993 & -0.049775 \\
4 & 1 & 0 & -3.092765 & -0.041039 & -0.367230 \\
5 & 1 & 0 & -2.303967 & -0.573619 & 0.992778 \\
6 & 1 & 0 & -1.853053 & -1.149265 & -0.487080 \\
7 & 1 & 0 & 1.311280 & 1.538364 & -1.078190 \\
8 & 1 & 0 & -1.251817 & 1.826920 & 0.365877 \\
9 & 1 & 0 & -0.750510 & 1.062047 & -1.240057 \\
10 & 17 & 0 & 0.880780 & -1.274201 & -0.071665 \\
11 & 1 & 0 & 0.521422 & 0.503840 & 1.540522 \\
12 & & 0 & & &
\end{tabular}

2-Bromoaziridine $\mathrm{E}=-2762.3053928$; Thermal Energy Correction $=0.104758$. Unique imaginary frequency $=-615.4095 \mathrm{~cm}^{-1}$.

\begin{tabular}{cccrrr}
$\begin{array}{c}\text { Center } \\
\text { Number }\end{array}$ & $\begin{array}{c}\text { Atomic } \\
\text { Number }\end{array}$ & $\begin{array}{c}\text { Atomic } \\
\text { Type }\end{array}$ & \multicolumn{2}{c}{ Coordinates (Angstroms) } \\
$-0--1$ & 0 & -2.666456 & 0.607443 & 0.276812 \\
1 & 1 & 0 & -1.706646 & 0.422355 & -0.191195 \\
2 & 6 & 0 & -1.679449 & 0.361725 & -1.272472 \\
3 & 1 & 0 & -0.525260 & 0.907006 & 0.480110 \\
4 & 6 & 0 & -1.849182 & -1.638848 & 0.018171 \\
5 & 7 & 0 & -0.640958 & 2.160640 & -0.053462 \\
6 & 7 & 0 & -0.497389 & 0.871990 & 1.568453 \\
7 & 1 & 0 & -2.390947 & -2.119760 & -0.703586 \\
8 & 1 & 0 & -2.194708 & -1.939667 & 0.931944 \\
9 & 1 & 0 & -0.867539 & -1.912265 & -0.065932 \\
10 & 1 & 0 & -0.553086 & 2.140204 & -1.079826 \\
11 & 1 & 0 & 1.190628 & -0.275382 & -0.032624 \\
12 & 35 & & & &
\end{tabular}


2,2-Dibromoaziridine $\mathrm{E}=-5334.6111682$; Thermal Energy Correction $=0.095178$. Unique imaginary frequency $=-659.5156 \mathrm{~cm}^{-1}$.

\begin{tabular}{cccccc}
$\begin{array}{c}\text { Center } \\
\text { Number }\end{array}$ & $\begin{array}{c}\text { Atomic } \\
\text { Number }\end{array}$ & $\begin{array}{c}\text { Atomic } \\
\text { Type }\end{array}$ & \multicolumn{2}{c}{$\begin{array}{c}\text { Coordinates } \\
\text { (Angstroms) }\end{array}$} \\
-1 & 1 & 0 & -0.816765 & 1.989388 & 1.412153 \\
-1 & 6 & 0 & 0.061033 & 1.569157 & 0.940462 \\
2 & 1 & 0 & 1.012086 & 2.080019 & 1.027336 \\
3 & 6 & 0 & 0.090840 & 0.133863 & 0.756988 \\
4 & 7 & 0 & -0.363385 & 2.390841 & -0.898168 \\
5 & 7 & 0 & 0.145184 & 0.008478 & 2.095411 \\
6 & 1 & 0 & -0.683446 & 3.362568 & -0.880199 \\
7 & 1 & 0 & -1.114952 & 1.805678 & -1.269844 \\
8 & 1 & 0 & 0.433742 & 2.327181 & -1.535363 \\
9 & 1 & 0 & 1.015704 & -0.413948 & 2.442307 \\
10 & 35 & 0 & -1.590822 & -0.626247 & -0.204968 \\
11 & 35 & 0 & 1.612816 & -0.464160 & -0.359655 \\
12 & & 0 & & &
\end{tabular}

Oxirane in THF $\mathrm{E}=-153.4672766$; Thermal Energy Correction $=0.061712$.

\begin{tabular}{cccrrr} 
Center & $\begin{array}{c}\text { Atomic } \\
\text { Number }\end{array}$ & $\begin{array}{c}\text { Atomic } \\
\text { Number }\end{array}$ & \multicolumn{3}{c}{ Coordinates (Angstroms) } \\
$-1 y p e$ & X & Y & $Z$ \\
-1 & 6 & 0 & 0.679206 & -0.470018 & 0.000000 \\
2 & 6 & 0 & 0.000000 & 0.825959 & 0.000000 \\
3 & 8 & 0 & -0.770705 & -0.403902 & 0.000000 \\
4 & 1 & 0 & 1.110746 & -0.848289 & 0.923028 \\
5 & 1 & 0 & 1.110746 & -0.848289 & -0.923028 \\
6 & 1 & 0 & -0.065545 & 1.396072 & 0.923024 \\
7 & 1 & 0 & -0.065545 & 1.396072 & -0.923024
\end{tabular}

Oxirane $+\mathrm{NH}_{3}$ Transition State in THF: $\mathrm{E}=-209.8667612$; Thermal Energy Correction $=$ 0.101355 . Unique imaginary frequency $=-664.2462 \mathrm{~cm}^{-1}$.

\begin{tabular}{|c|c|c|c|c|c|}
\hline \multirow{2}{*}{$\begin{array}{l}\text { Center } \\
\text { Number }\end{array}$} & \multirow{2}{*}{$\begin{array}{l}\text { Atomic } \\
\text { Number }\end{array}$} & \multirow{2}{*}{$\begin{array}{l}\text { Atomic } \\
\text { Type }\end{array}$} & \multicolumn{3}{|c|}{ Coordinates (Angstroms) } \\
\hline & & & $\mathrm{X}$ & $\mathrm{Y}$ & Z \\
\hline 1 & 6 & 0 & 0.000000 & 0.492122 & 0.000000 \\
\hline 2 & 7 & 0 & -1.965614 & 0.134540 & 0.000000 \\
\hline 3 & 6 & 0 & 0.765228 & -0.749106 & 0.000000 \\
\hline 4 & 1 & 0 & -0.041902 & 1.048876 & 0.925770 \\
\hline 5 & 1 & 0 & -0.041902 & 1.048876 & -0.925770 \\
\hline 6 & 8 & 0 & 1.863907 & 0.131714 & 0.000000 \\
\hline 7 & 1 & 0 & -2.515838 & 0.999549 & 0.000000 \\
\hline 8 & 1 & 0 & 0.662277 & -1.368971 & 0.901214 \\
\hline 9 & 1 & 0 & 0.662277 & -1.368971 & -0.901214 \\
\hline 10 & 1 & 0 & -2.234120 & -0.406472 & -0.827359 \\
\hline 11 & 1 & 0 & -2.234120 & -0.406472 & 0.827359 \\
\hline
\end{tabular}




\begin{tabular}{|c|c|c|c|c|c|c|c|c|c|c|c|c|c|}
\hline \multicolumn{14}{|c|}{ NBO Charges } \\
\hline & $\mathrm{C}_{\mathrm{R}}$ & $X_{1}$ & $X_{2}$ & $\mathbf{C}_{\mathrm{v}}$ & $\mathbf{X}_{3}$ & $X_{4}$ & $X_{5}$ & $\left(C_{R) \text { eff }}\right.$ & $\mathbf{N}_{\mathbf{L}}$ & $\underline{\mathrm{H}}-\mathrm{N}_{\mathrm{L}}$ & $\left(N_{L}\right)_{\text {eff }}$ & $\begin{array}{c}\mathbf{d}_{\mathrm{CR}-\mathrm{NH}} \\
\hat{A}\end{array}$ & $\begin{array}{c}\mathrm{E} \\
\mathrm{kcal} / \mathrm{mol}\end{array}$ \\
\hline 4 & -0.304 & 0.264 & 0.248 & -0.309 & 0.179 & 0.160 & & 0.237 & -1.089 & 0.351 & -0.738 & 2.271 & -25.6 \\
\hline 5 & -0.274 & 0.276 & 0.252 & 0.186 & -0.498 & 0.199 & & 0.141 & -0.815 & 0.379 & -0.436 & 2.026 & -10.1 \\
\hline 6 & -0.316 & 0.277 & 0.264 & 0.754 & -0.432 & -0.415 & & 0.132 & -0.854 & 0.396 & -0.459 & 2.072 & -9.7 \\
\hline 7 & -0.121 & 0.262 & 0.255 & 0.010 & 0.254 & -0.387 & & 0.273 & -0.881 & 0.356 & -0.525 & 2.104 & -22.6 \\
\hline 8 & -0.004 & 0.219 & 0.203 & 0.167 & -0.210 & -0.089 & & 0.286 & -0.905 & 0.359 & -0.547 & 1.991 & -26.1 \\
\hline 9 & 0.012 & 0.198 & 0.195 & 0.043 & 0.190 & -0.374 & & 0.265 & -0.810 & 0.317 & -0.493 & 2.082 & -20.8 \\
\hline 10 & -0.004 & 0.220 & 0.207 & 0.055 & -0.173 & -0.021 & & 0.284 & -0.870 & 0.366 & -0.504 & 1.961 & -24.2 \\
\hline 11 & -0.084 & 0.266 & 0.301 & -0.461 & 0.274 & 0.284 & -0.087 & 0.492 & -0.719 & 0.416 & -0.303 & 1.964 & -25.2 \\
\hline 13 & -0.200 & 0.258 & 0.304 & 0.039 & 0.235 & 0.239 & -0.505 & 0.370 & -1.075 & 0.361 & -0.715 & 2.192 & -40.1 \\
\hline 15 & 0.367 & -0.349 & 0.256 & 0.148 & -0.495 & 0.211 & & 0.138 & -0.800 & 0.385 & -0.415 & 2.006 & -9.5 \\
\hline 16 & 0.393 & -0.332 & 0.234 & 0.155 & 0.200 & -0.487 & & 0.164 & -0.821 & 0.379 & -0.442 & 2.052 & -11.7 \\
\hline 17 & 0.023 & 0.033 & 0.307 & 0.000 & -0.362 & 0.247 & & 0.248 & -0.882 & 0.383 & -0.500 & 1.998 & -20.6 \\
\hline 18 & 0.045 & 0.080 & 0.280 & -0.012 & 0.237 & -0.341 & & 0.287 & -0.903 & 0.377 & -0.526 & 2.049 & -24.5 \\
\hline
\end{tabular}

